Pacific

Journal of

Mathematics

GEOMETRIC PROPERTIES OF JULIA SETS OF THE COMPOSITION OF POLYNOMIALS OF THE FORM $z^{2}+c_{n}$

RAINER BRÜCK

Volume 198 No. 2

April 2001 


\title{
GEOMETRIC PROPERTIES OF JULIA SETS OF THE COMPOSITION OF POLYNOMIALS OF THE FORM $z^{2}+c_{n}$
}

\author{
RAINER BRÜCK
}

\begin{abstract}
For a sequence $\left(c_{n}\right)$ of complex numbers we consider the quadratic polynomials $f_{c_{n}}(z):=z^{2}+c_{n}$ and the sequence $\left(F_{n}\right)$ of iterates $F_{n}:=f_{c_{n}} \circ \cdots \circ f_{c_{1}}$. The Fatou set $\mathcal{F}_{\left(c_{n}\right)}$ is by definition the set of all $z \in \widehat{\mathbb{C}}$ such that $\left(F_{n}\right)$ is normal in some neighbourhood of $z$, while the complement of $\mathcal{F}_{\left(c_{n}\right)}$ is called the Julia set $\mathcal{J}_{\left(c_{n}\right)}$. The aim of this article is to study geometric properties, Lebesgue measure and Hausdorff dimension of the Julia set $\mathcal{J}_{\left(c_{n}\right)}$ provided that the sequence $\left(c_{n}\right)$ is bounded.
\end{abstract}

\section{Introduction.}

For a sequence $\left(c_{n}\right)$ of complex numbers we consider the quadratic polynomials $f_{c_{n}}(z):=z^{2}+c_{n}$ and the sequence $\left(F_{n}\right)$ of iterates $F_{n}:=f_{c_{n}} \circ \cdots \circ f_{c_{1}}$. (Note that $F_{n}$ depends on $c_{1}, \ldots, c_{n}$ which we do not indicate explicitly in the notation.) If $c_{n}=c$ for all $n$, we write $f_{c}^{n}$ instead of $F_{n}$. The Fatou set $\mathcal{F}_{\left(c_{n}\right)}$ is by definition the set of all $z \in \widehat{\mathbb{C}}:=\mathbb{C} \cup\{\infty\}$ such that $\left(F_{n}\right)$ is normal (in the sense of Montel) in some neighbourhood of $z$, while the complement of $\mathcal{F}_{\left(c_{n}\right)}$ (in $\widehat{\mathbb{C}}$ ) is called the Julia set $\mathcal{J}_{\left(c_{n}\right)}$. A component of the Fatou set is called a stable domain. For iteration theory of a fixed function we refer the reader to the books of Beardon [Be], Carleson and Gamelin $[\mathbf{C G}]$, Milnor $[\mathbf{M}]$ or Steinmetz [St]. We also mention the survey articles of Blanchard [B1], Lyubich [L2] or Eremenko and Lyubich [EL].

We always assume that $\left|c_{n}\right| \leq \delta$ for some $\delta>0$. Then from [B̈̈2] it is known that to some extent the sequence $\left(F_{n}\right)$ behaves similar to the sequence $\left(f_{c}^{n}\right)$. There exists a stable domain $\mathcal{A}_{\left(c_{n}\right)}(\infty)$ which contains the point $\infty$ and wherein $F_{n} \rightarrow \infty$ as $n \rightarrow \infty$ locally uniformly. This domain need not be invariant (i.e., $f_{c_{k}}\left(\mathcal{A}_{\left(c_{n}\right)}(\infty)\right) \subset \mathcal{A}_{\left(c_{n}\right)}(\infty)$ for all $k$ ) or backward invariant (i.e., $f_{c_{k}}^{-1}\left(\mathcal{A}_{\left(c_{n}\right)}(\infty)\right) \subset \mathcal{A}_{\left(c_{n}\right)}(\infty)$ for all $k$ ), but there exists an invariant domain $M=M_{\delta} \subset \mathcal{A}_{\left(c_{n}\right)}(\infty)$ which contains the point $\infty$ and which satisfies $\mathcal{A}_{\left(c_{n}\right)}(\infty)=\left\{z \in \widehat{\mathbb{C}}: F_{k}(z) \in M\right.$ for some $\left.k \in \mathbb{N}\right\}$. Therefore, the filled Julia set $\mathcal{K}_{\left(c_{n}\right)}:=\widehat{\mathbb{C}} \backslash \mathcal{A}_{\left(c_{n}\right)}(\infty)$ and the Julia set $\mathcal{J}_{\left(c_{n}\right)}$ are compact in $\mathbb{C}$, and $\mathcal{K}_{\left(c_{n}\right)}$ is the set of all $z \in \mathbb{C}$ such that $\left(F_{k}(z)\right)_{k=1}^{\infty}$ is bounded. Furthermore, we have $\mathcal{J}_{\left(c_{n}\right)}=\partial \mathcal{A}_{\left(c_{n}\right)}(\infty)=\partial \mathcal{K}_{\left(c_{n}\right)}$. Also $\mathcal{J}_{\left(c_{n}\right)}$ and $\mathcal{K}_{\left(c_{n}\right)}$ are perfect sets. 
Finally, $\mathcal{J}_{\left(c_{n}\right)}$ and $\mathcal{F}_{\left(c_{n}\right)}$ are invariant in the sense that $F_{k}^{-1}\left(F_{k}\left(\mathcal{J}_{\left(c_{n}\right)}\right)\right)=\mathcal{J}_{\left(c_{n}\right)}$ and $F_{k}^{-1}\left(F_{k}\left(\mathcal{F}_{\left(c_{n}\right)}\right)\right)=\mathcal{F}_{\left(c_{n}\right)}$ for all $k \in \mathbb{N}$. For further results we also refer to $[\mathbf{B r u ̈}],[\mathbf{B B R}],[\mathbf{B u ̈ 1}]$ and $[\mathbf{F S}]$.

The Mandelbrot set $\mathcal{M}$ is defined as the set of all $c \in \mathbb{C}$ such that $\left(f_{c}^{n}(0)\right)_{n=1}^{\infty}$ is bounded, and $\mathcal{M}$ is compact in $\mathbb{C}$. It plays an important role in iteration of a fixed quadratic polynomial $f_{c}$. We recall that the largest disk with center 0 which is contained in $\mathcal{M}$ has radius $\frac{1}{4}$.

The plan of this article is as follows. After introducing some notations and known auxiliary results (Section 2) we show that the Julia set $\mathcal{J}_{\left(c_{n}\right)}$ is always uniformly perfect (Section 3 ).

Our main result (Section 4) states that the Julia set $\mathcal{J}_{\left(c_{n}\right)}$ is a quasicircle provided that $\left|c_{n}\right| \leq \delta$ for some $\delta<\frac{1}{4}$. This is done by proving that $\mathcal{F}_{\left(c_{n}\right)}$ consists of two simply connected John domains $\mathcal{A}_{\left(c_{n}\right)}(0)$ and $\mathcal{A}_{\left(c_{n}\right)}(\infty)$ which have $\mathcal{J}_{\left(c_{n}\right)}$ as their common boundaries.

Concerning the two-dimensional Lebesgue measure $m_{2}\left(\mathcal{J}_{\left(c_{n}\right)}\right)$ of Julia sets (Section 5) we show that it is almost surely zero provided that the $c_{n}$ are randomly chosen in $\{z \in \mathbb{C}:|z| \leq \delta\}$ for some $\delta>\frac{1}{4}$. For $\delta<\frac{1}{4}$ we always have $m_{2}\left(\mathcal{J}_{\left(c_{n}\right)}\right)=0$.

Section 6 deals with Hausdorff dimension $\operatorname{dim}_{\mathrm{H}} \mathcal{\partial}_{\left(c_{n}\right)}$ of Julia sets. We give a lower estimate for $\operatorname{dim}_{\mathrm{H}} \partial_{\left(c_{n}\right)}$ depending only on $\delta$ which implies that $\operatorname{dim}_{\mathrm{H}} \mathcal{J}_{\left(c_{n}\right)}$ is always positive. For that purpose we prove that the Green function of $\mathcal{A}_{\left(c_{n}\right)}(\infty)$ (which is known to exist) is Hölder continuous. Furthermore, for $\delta<\frac{1}{4}$ it follows that $\operatorname{dim}_{\mathrm{H}} \mathcal{J}_{\left(c_{n}\right)}<2$.

A point $\zeta \in \mathbb{C}$ is called a repelling fixpoint of the sequence of iterates $\left(F_{n}\right)$ if $F_{k}(\zeta)=\zeta$ for some $k \in \mathbb{N}$ and $\left|F_{k}^{\prime}(\zeta)\right|>1$. The set of all those points is denoted by $\mathcal{R}_{\left(c_{n}\right)}$. In this general setting it is not necessarily true that $\mathcal{R}_{\left(c_{n}\right)} \subset \mathcal{J}_{\left(c_{n}\right)}$. But we prove (Section 7) that if $\left|c_{n}\right| \leq \delta<\frac{1}{4}$, then the derived set of $\mathcal{R}_{\left(c_{n}\right)}$ coincides with $\mathcal{J}_{\left(c_{n}\right)}$. In the last section we investigate the asymptotic distribution of certain predecessors.

\section{Notations and auxiliary results.}

We introduce a few further notations and collect some known auxiliary results that are frequently used in the sequel. If $E \subset \mathbb{C}$, then $E^{\prime}$ denotes the derived set (that is the set of points $z \in \mathbb{C}$ such that every neighbourhood of $z$ contains a point $w \in E \backslash\{z\}), \bar{E}$ the closure and $E^{\circ}$ the set of interior points of $E$. Furthermore, the diameter of $E$ is defined by $\operatorname{diam} E:=\sup \{|z-w|: z, w \in E\}$, and the distance of a point $z \in \mathbb{C}$ from $E$ by $\operatorname{dist}(z, E):=\inf \{|z-w|: w \in E\}$. For $a \in \mathbb{C}$ and $r>0$ we set $D_{r}(a):=\{z \in \mathbb{C}:|z-a|<r\}, D_{r}:=D_{r}(0), \mathbb{D}:=D_{1}$ and $K_{r}:=\bar{D}_{r}$. Finally, for $R>0$ let $\Delta_{R}:=\{z \in \widehat{\mathbb{C}}:|z|>R\}$. 
If $\left(c_{n}\right) \in K_{\delta}^{\mathbb{N}}$, then the invariant domain $M \subset \mathcal{A}_{\left(c_{n}\right)}(\infty)$ may be chosen as $M=\Delta_{R}$ for any

$$
R \geq R_{\delta}:=\frac{1}{2}(1+\sqrt{1+4 \delta}) .
$$

More precisely, if $R>R_{\delta}$, then $f_{c}\left(\Delta_{R_{\delta}}\right) \subset \Delta_{R_{\delta}}$ and $f_{c}\left(\bar{\Delta}_{R}\right) \subset \Delta_{R}$ for all $c \in K_{\delta}$. This implies that $\mathcal{K}_{\left(c_{n}\right)} \subset K_{R_{\delta}}$. If $\delta \leq \frac{1}{4}$, we set

$$
r_{\delta}:=\frac{1}{2}(1+\sqrt{1-4 \delta}) \in\left[\frac{1}{2}, 1\right], \quad s_{\delta}:=\frac{1}{2}(1-\sqrt{1-4 \delta}) \in\left[0, \frac{1}{2}\right] .
$$

Then we have $f_{c}\left(D_{s_{\delta}}\right) \subset D_{s_{\delta}}, f_{c}\left(D_{r_{\delta}}\right) \subset D_{r_{\delta}}$ and $f_{c}\left(\bar{D}_{r}\right) \subset D_{r}$ for all $c \in K_{\delta}$ and all $r \in\left(s_{\delta}, r_{\delta}\right)$. This implies that there exists a stable domain $\mathcal{A}_{\left(c_{n}\right)}(0) \supset D_{r_{\delta}}$, and there holds $\mathcal{J}_{\left(c_{n}\right)} \subset K_{R_{\delta}} \cap \bar{\Delta}_{r_{\delta}}$.

From [FS, Theorem 2.1] it follows that $\mathcal{A}_{\left(c_{n}\right)}(\infty)$ is regular for logarithmic potential theory which means that the Green function of $\mathcal{A}_{\left(c_{n}\right)}(\infty)$ with pole at infinity exists. More precisely, the function $g_{\left(c_{n}\right)}$ defined by

$$
g_{\left(c_{n}\right)}(z):=\lim _{k \rightarrow \infty} \frac{1}{2^{k}} \log ^{+}\left|F_{k}(z)\right|
$$

is continuous in $\mathbb{C}, g_{\left(c_{n}\right)}(z)=0$ for $z \in \mathcal{K}_{\left(c_{n}\right)}$, and it is the Green function of $\mathcal{A}_{\left(c_{n}\right)}(\infty)$ with pole at infinity.

Furthermore, we introduce the critical set (or set of critical points)

$$
\mathcal{C}_{\left(c_{n}\right)}:=\left\{z \in \mathbb{C}: F_{j}(z)=0 \text { for some } j \in \mathbb{N}_{0}\right\}
$$

of $\left(F_{n}\right)$, where $F_{0}(z):=z$. This is motivated by the fact that

$$
F_{k}^{\prime}(z)=2^{k} \prod_{j=0}^{k-1} F_{j}(z)
$$

so that $F_{k}^{\prime}(z)=0$ if and only if $F_{j}(z)=0$ for some $j \in\{0,1, \ldots, k-1\}$. We call a point $w \in \mathbb{C}$ a critical value of $\left(F_{n}\right)$, if $w=F_{k}(z)$ and $F_{k}^{\prime}(z)=0$ for some $k \in \mathbb{N}$ and some $z \in \mathbb{C}$. If $w \in \mathbb{C}$ is not a critical value of $F_{k}$, then in some sufficiently small disk $D_{\varepsilon}(w)$ there exist $2^{k}$ analytic branches of the inverse function of $F_{k}$.

Finally, we recall a result of Büger [Bü1] that the Julia set $\mathcal{J}_{\left(c_{n}\right)}$ is selfsimilar. This means that for any open set $D$ meeting $\mathcal{J}_{\left(c_{n}\right)}$ there exists $k_{0} \in \mathbb{N}$ such that $F_{k}\left(\mathcal{J}_{\left(c_{n}\right)} \cap D\right)=F_{k}\left(\mathcal{J}_{\left(c_{n}\right)}\right)$ for all $k \geq k_{0}$.

\section{Uniform perfectness of Julia sets.}

An open set $A \subset \widehat{\mathbb{C}}$ is called a conformal annulus, if it can be mapped conformally onto an annulus $\{z \in \mathbb{C}: 1<|z|<\varrho\}$ for some $\varrho>1$. Then the number $\varrho$ is uniquely determined and $\bmod A:=\frac{1}{2 \pi} \log \varrho$ is called the modulus of $A$. Now, let $E \subset \widehat{\mathbb{C}}$ be a compact set. A conformal annulus $A$ seperates $E$, if both components of $\widehat{\mathbb{C}} \backslash A$ meet $E$. The set $E$ is called uniformly perfect, if it is not a single point and if there is a constant $\alpha>0$ such that for any 
conformal annulus $A$ which seperates $E$ there holds $\bmod A \leq \alpha$. Obviously, a uniformly perfect set is also perfect (that is $E^{\prime}=E$ ), and every connected compact set with at least two points is uniformly perfect. Uniformly perfect sets were introduced by Beardon and Pommerenke $[\mathbf{B e P}]$ (see also $[\mathbf{P 1}]$ ). It is known that the Julia set of a fixed rational function is always uniformly perfect $[\mathbf{M R}]$ (see also [CG, p. 64]). We show that this result extends to our situation.

Theorem 3.1. Let $\delta>0$ and $\left(c_{n}\right) \in K_{\delta}^{\mathbb{N}}$. Then the Julia set $\mathcal{J}_{\left(c_{n}\right)}$ is uniformly perfect.

Proof. We assume that $\mathcal{J}_{\left(c_{n}\right)}$ is not uniformly perfect. Then there exists a sequence of conformal annuli $A_{k} \subset \mathcal{F}_{\left(c_{n}\right)}$ which seperate $\mathcal{J}_{\left(c_{n}\right)}$ and $\bmod A_{k} \rightarrow$ $\infty$ as $k \rightarrow \infty$. Let $E_{k}$ be the component of $\widehat{\mathbb{C}} \backslash A_{k}$ with the smaller chordal diameter (which we denote by $\operatorname{diam}_{\chi} E_{k}$ ). Then we have $\operatorname{diam}_{\chi} E_{k} \rightarrow 0$ as $k \rightarrow \infty$. If $\lambda_{k}: \mathbb{D} \rightarrow A_{k} \cup E_{k}$ is a conformal map of $\mathbb{D}$ onto $A_{k} \cup E_{k}$ with $\lambda_{k}(0) \in E_{k}$, and if $M_{k}:=\lambda_{k}^{-1}\left(E_{k}\right) \subset \mathbb{D}$, then $M_{k}$ is compact and connected, $0 \in M_{k}$ and $\operatorname{diam}_{\chi} M_{k} \rightarrow 0$ as $k \rightarrow \infty$.

It is elementary to see that $\left(f_{c_{n}}\right)$ satisfies a uniform Lipschitz condition with respect to the chordal metric $\chi$, that means that there exists a constant $L>0$ (which depends only on $\delta$ but not on $n$ ) such that $\chi\left(f_{c_{n}}(z), f_{c_{n}}(w)\right) \leq$ $L \chi(z, w)$ for all $z, w \in \widehat{\mathbb{C}}$ and all $n \in \mathbb{N}$. From Lemma 4.1 in [BBR] we know that $\operatorname{diam} F_{k}\left(\mathcal{J}_{\left(c_{n}\right)}\right) \geq 1$ for all $k \in \mathbb{N}_{0}$ so that $\operatorname{diam}_{\chi} F_{k}\left(\mathcal{J}_{\left(c_{n}\right)}\right) \geq C:=$ $2\left(1+R_{\delta}^{2}\right)^{-1}$.

We choose $\varepsilon>0$ with $\varepsilon<C$ and

$$
\frac{C}{3}>L \varepsilon \text {. }
$$

Let $k_{0} \in \mathbb{N}$ such that $\operatorname{diam}_{\chi} E_{k}<\varepsilon$ for all $k \geq k_{0}$. Since $\left(A_{k} \cup E_{k}\right) \cap \mathcal{J}_{\left(c_{n}\right)} \neq \varnothing$ and since $\mathcal{J}_{\left(c_{n}\right)}$ is self-similar (cf. [Bü1]), for every $k \geq k_{0}$ there exists a smallest index $m(k) \in \mathbb{N}$ such that $\operatorname{diam}_{\chi} F_{m(k)}\left(E_{k}\right)>\varepsilon$. Setting $G_{k}:=$ $F_{m(k)} \circ \lambda_{k}$ we obtain

$$
\operatorname{diam}_{\chi} G_{k}\left(M_{k}\right)>\varepsilon
$$

for all $k \geq k_{0}$. By the choice of $m(k)$ we have $\operatorname{diam}_{\chi} F_{m(k)-1}\left(E_{k}\right) \leq \varepsilon$ and thus diam $F_{m(k)}\left(E_{k}\right)=\operatorname{diam}_{\chi} f_{c_{m(k)}}\left(F_{m(k)-1}\left(E_{k}\right)\right) \leq L \varepsilon$ for all $k \geq k_{0}$.

Because of (3.1) there exist at least three different points $a_{1, k}, a_{2, k}, a_{3, k} \in$ $F_{m(k)}\left(\mathcal{J}_{\left(c_{n}\right)}\right)$ whose chordal distance is greater than $L \varepsilon$. We have $G_{k}(\mathbb{D} \backslash$ $\left.M_{k}\right)=F_{m(k)}\left(\lambda_{k}\left(\mathbb{D} \backslash M_{k}\right)\right)=F_{m(k)}\left(A_{k}\right) \subset F_{m(k)}\left(\mathcal{F}_{\left(c_{n}\right)}\right)$ and $\operatorname{diam}_{\chi} G_{k}\left(M_{k}\right)=$ $\operatorname{diam}_{\chi} F_{m(k)}\left(E_{k}\right) \leq L \varepsilon$ for all $k \geq k_{0}$. This implies that $G_{k}$ omits at least two of the values $a_{1, k}, a_{2, k}, a_{3, k}$ in $\mathbb{D}$ and hence $\left(G_{k}\right)$ is normal in $\mathbb{D}$ by a generalized version of Montel's theorem (cf. [Be, p. 57]). Since diam ${ }_{\chi} M_{k} \rightarrow$ 0 as $k \rightarrow \infty$ and $0 \in M_{k}$ we get $\operatorname{diam}_{\chi} G_{k}\left(M_{k}\right) \rightarrow 0$ as $k \rightarrow \infty$ which contradicts (3.2). 


\section{Julia sets and quasicircles.}

From iteration theory of a fixed function it is known that $\mathcal{J}\left(f_{c}\right)$ is a quasicircle if $c$ is in the interior of the main cardioid of the Mandelbrot set (cf. Yakobson $[\mathbf{Y}]$, see also [CG, p. 103]). The goal of this section is to show that this result remains valid in our general situation provided that $\delta<\frac{1}{4}$. We do this in several steps, and we first recall some facts on quasicircles and John domains.

A quasicircle $\Gamma \subset \mathbb{C}$ is the image of the unit circle $\partial \mathbb{D}$ under a quasiconformal homeomorphism of $\mathbb{C}$ onto itself. An equivalent geometric definition is the three-point property, i.e., there exists a constant $a>0$ such that if $z_{1}$, $z_{2}, z_{3} \in \Gamma$ and $z_{2}$ is on the arc between $z_{1}$ and $z_{3}$ with the smaller diameter, then $\left|z_{1}-z_{2}\right|+\left|z_{2}-z_{3}\right| \leq a\left|z_{1}-z_{3}\right|$. A quasicircle may be non-rectifiable but it has no cusps. For details we refer, for example, to the books of Ahlfors $[\mathbf{A}]$ or Lehto and Virtanen $[\mathbf{L V}]$.

A domain $G \subset \widehat{\mathbb{C}}$ with $\partial G \subset \mathbb{C}$ is called a John domain, if there exists a constant $b>0$ and a point $w_{0} \in G$ such that for any $z_{0} \in G$, there is an arc $\gamma=\gamma\left(z_{0}\right) \subset G$ joining $z_{0}$ and $w_{0}$ and satisfying $\operatorname{dist}(z, \partial G) \geq b\left|z-z_{0}\right|$ for all $z \in \gamma$. A simply connected John domain $G$ has locally connected boundary $\partial G$ so that by Carathéodory's theorem (cf. [P2, p. 20]) the Riemann map from $\mathbb{D}$ onto $G$ extends continuously to $\overline{\mathbb{D}}$. The image of a John domain under a quasiconformal homeomorphism of $\widehat{\mathbb{C}}$ onto itself is again a John domain. Thus, the two complementary domains of a quasicircle are John domains. Conversely, if the two complementary components of a Jordan curve (a homeomorphic image of the unit circle) $\Gamma$ are John domains, then $\Gamma$ is a quasicircle. For this and further background material we refer to $[\mathbf{N V}]$.

For $\delta \leq \frac{1}{4}$ we know that $\mathcal{J}_{\left(c_{n}\right)}$ is connected [BBR], and since $\mathcal{J}_{\left(c_{n}\right)}=$ $\partial \mathcal{A}_{\left(c_{n}\right)}(\infty)$, the stable domain $\mathcal{A}_{\left(c_{n}\right)}(\infty)$ is simply connected. Furthermore, there exists a stable domain $\mathcal{A}_{\left(c_{n}\right)}(0)$ containing $D_{r_{\delta}}$. We now show:

Theorem 4.1. Let $\delta \leq \frac{1}{4},\left(c_{n}\right) \in K_{\delta}^{\mathbb{N}}$ and $s_{\delta} \leq r \leq r_{\delta}$. Then there holds $\mathcal{A}_{\left(c_{n}\right)}(0)=\bigcup_{k=0}^{\infty} F_{k}^{-1}\left(D_{r}\right)$ and $\partial \mathcal{A}_{\left(c_{n}\right)}(0)=\mathcal{J}_{\left(c_{n}\right)}$. In particular, $\mathcal{A}_{\left(c_{n}\right)}(0)$ is simply connected and $\mathcal{F}_{\left(c_{n}\right)}=\mathcal{A}_{\left(c_{n}\right)}(0) \cup \mathcal{A}_{\left(c_{n}\right)}(\infty)$.

Proof. We set $A:=\bigcup_{k=0}^{\infty} U_{k}$ with $U_{k}:=F_{k}^{-1}\left(D_{r}\right)$. It is elementary to see that each $U_{k}$ is a domain containing $D_{r}$, and since $D_{r}$ is invariant, we get $U_{k} \subset \mathcal{F}_{\left(c_{n}\right)}$. Thus, $A$ is a domain with $D_{r} \subset A \subset \mathcal{F}_{\left(c_{n}\right)}$ which gives $A \subset \mathcal{A}_{\left(c_{n}\right)}(0)$.

We show that $\mathcal{J}_{\left(c_{n}\right)} \subset \partial A$. For that purpose, let $z_{0} \in \mathcal{J}_{\left(c_{n}\right)}$ and $D:=$ $D_{\varepsilon}\left(z_{0}\right)$ for $\varepsilon>0$. By Montel's theorem the set $\widehat{\mathbb{C}} \backslash \bigcup_{k=0}^{\infty} F_{k}(D)$ contains at most two points so that there exists $w \in D_{r}$ such that $w \in F_{m}(D)$ for some $m \in \mathbb{N}_{0}$. Therefore, $D_{r} \cap F_{m}(D)$ is a non-empty open set, and this 
implies that there exists $\zeta \in D \backslash \mathcal{J}_{\left(c_{n}\right)}$ with $F_{m}(\zeta) \in D_{r}$. That means $\zeta \in A$, and since $\varepsilon>0$ was arbitrary we arrive at $z_{0} \in \partial A$. Summarizing, we have $A \subset \mathcal{A}_{\left(c_{n}\right)}(0)$ and $\partial \mathcal{A}_{\left(c_{n}\right)}(0) \subset \mathcal{J}_{\left(c_{n}\right)} \subset \partial A$ which gives the assertion.

For $\delta<\frac{1}{4}$ and $\frac{1}{2}<r<r_{\delta}$ we set $V:=\Delta_{r} \supset \mathcal{J}_{\left(c_{n}\right)}$. Then $V$ is backward invariant, and $V$ does not contain any critical value of $\left(F_{n}\right)$ so that in every disk $D \subset V$ there exist $2^{n}$ analytic branches $F_{n}^{-1}$ of the inverse function of $F_{n}$. We prove:

Lemma 4.2. Let $\delta<\frac{1}{4},\left(c_{n}\right) \in K_{\delta}^{\mathbb{N}}$ and $\frac{1}{2}<r<r_{\delta}$. Furthermore, let $\gamma:[0,1] \rightarrow V$ be a rectifiable curve in $V:=\Delta_{r}, z:=\gamma(0), w:=\gamma(1)$ and let $F_{n}^{-1}$ be an analytic branch of the inverse function of $F_{n}$ in some disk $D \subset V$ with center $z$. Finally, we denote the analytic continuation of $F_{n}^{-1}$ along $\gamma$ also by $F_{n}^{-1}$. Then there holds

$$
\left|\frac{\left(F_{n}^{-1}\right)^{\prime}(z)}{\left(F_{n}^{-1}\right)^{\prime}(w)}\right| \leq 1+\alpha \ell(\gamma) e^{\alpha \ell(\gamma)},
$$

where $\alpha:=4 r(2 r-1)^{-1}$ and $\ell(\gamma)$ denotes the length of $\gamma$. In particular, for any disk $D \subset V$ and any analytic branch $F_{n}^{-1}$ in $D$ there holds

$$
\left|\frac{\left(F_{n}^{-1}\right)^{\prime}(z)}{\left(F_{n}^{-1}\right)^{\prime}(w)}\right| \leq 1+\alpha e^{\alpha d}|z-w|
$$

for all $z, w \in D$ and $n \in \mathbb{N}$, where $d:=\operatorname{diam} D$.

Proof. For $n \in \mathbb{N}$ and $k=0,1, \ldots, n-1$ we set $F_{n, k}:=f_{c_{n}} \circ \cdots \circ f_{c_{k+1}}$. Since

$$
\left(F_{n}^{-1}\right)^{\prime}(z)=\frac{1}{F_{n}^{\prime}\left(F_{n}^{-1}(z)\right)}=\frac{1}{2^{n} \prod_{j=0}^{n-1} F_{j}\left(F_{n}^{-1}(z)\right)}=\frac{1}{2^{n} \prod_{j=0}^{n-1} F_{n, j}^{-1}(z)}
$$

and $V$ is backward invariant we have

$$
\left|\left(F_{n}^{-1}\right)^{\prime}(z)\right| \leq q^{n} \quad(z \in V),
$$

or

$$
\left|\left(F_{n, k}^{-1}\right)^{\prime}(z)\right| \leq q^{n-k} \quad(z \in V),
$$

where $q:=\frac{1}{2 r}<1$. This implies

$$
\left|F_{n, k}^{-1}(w)-F_{n, k}^{-1}(z)\right| \leq\left|\int_{z}^{w}\right|\left(F_{n, k}^{-1}\right)^{\prime}(\zeta)|| d \zeta|| \leq q^{n-k} \ell(\gamma),
$$

where we integrate over the curve $\gamma$. Furthermore, we have

$$
\frac{\left(F_{n}^{-1}\right)^{\prime}(z)}{\left(F_{n}^{-1}\right)^{\prime}(w)}=\prod_{k=0}^{n-1} \frac{F_{k}\left(F_{n}^{-1}(w)\right)}{F_{k}\left(F_{n}^{-1}(z)\right)}=\prod_{k=0}^{n-1} \frac{F_{n, k}^{-1}(w)}{F_{n, k}^{-1}(z)} .
$$


Writing

$$
\frac{F_{n, k}^{-1}(w)}{F_{n, k}^{-1}(z)}=1+\frac{F_{n, k}^{-1}(w)-F_{n, k}^{-1}(z)}{F_{n, k}^{-1}(z)},
$$

we obtain from (4.1)

$$
\left|\frac{F_{n, k}^{-1}(w)}{F_{n, k}^{-1}(z)}\right| \leq 1+2 q^{n-k+1} \ell(\gamma) .
$$

This implies

$$
\begin{aligned}
\left|\frac{\left(F_{n}^{-1}\right)^{\prime}(z)}{\left(F_{n}^{-1}\right)^{\prime}(w)}\right| & \leq \prod_{k=0}^{n-1}\left(1+2 q^{n-k+1} \ell(\gamma)\right)=\prod_{k=2}^{n+1}\left(1+2 q^{k} \ell(\gamma)\right) \\
& \leq \prod_{k=0}^{\infty}\left(1+2 q^{k} \ell(\gamma)\right)=\exp \left(\sum_{k=0}^{\infty} \log \left(1+2 q^{k} \ell(\gamma)\right)\right) \\
& \leq \exp \left(\sum_{k=0}^{\infty} 2 q^{k} \ell(\gamma)\right)=e^{\alpha \ell(\gamma)},
\end{aligned}
$$

where $\alpha:=2(1-q)^{-1}$. Finally, this gives the assertion since $e^{x} \leq 1+x e^{x}$ for $x \geq 0$.

Theorem 4.3. Let $\delta<\frac{1}{4}$ and $\left(c_{n}\right) \in K_{\delta}^{\mathbb{N}}$. Then $\mathcal{A}_{\left(c_{n}\right)}(\infty)$ is a John domain.

Proof. We first introduce a few notations. For $z_{1}, z_{2} \in \mathbb{C}$ let $\left[z_{1}, z_{2}\right]$ denote the line segment joining $z_{1}$ and $z_{2}$. If $\zeta \in \mathbb{C}, \zeta \neq 0$, and if $\Gamma$ is the ray from 0 to $\infty$ passing through $\zeta$, then let $\Gamma_{\zeta}$ denote that part of $\Gamma$ from $\zeta$ to $\infty$.

Let $R>R_{\delta}$ such that $R^{2}+\delta-R \leq \frac{1}{2}, \varepsilon:=R-R_{\delta} \leq 1$ and $U_{k}:=F_{k}^{-1}\left(\Delta_{R}\right)$ for $k \in \mathbb{N}$. Then we have $U_{k} \subset U_{k+1} \subset \mathcal{A}_{\left(c_{n}\right)}(\infty)$ and $\mathcal{A}_{\left(c_{n}\right)}(\infty)=\bigcup_{k=1}^{\infty} U_{k}$. Furthermore, $U_{k}$ is a simply connected domain (in $\widehat{\mathbb{C}}$ ) bounded by an analytic Jordan curve. For $z \in \mathcal{A}_{\left(c_{n}\right)}(\infty)$ let $d(z):=\operatorname{dist}\left(z, \mathcal{J}_{\left(c_{n}\right)}\right)$. We prove a lower estimate for $d(z)$, if $z \in U_{k}$ for some $k \in \mathbb{N}$. We set $w:=F_{k}(z)$. If $U$ denotes the component of $F_{k}^{-1}\left(D_{\varepsilon}(w)\right)$ containing $z$, there holds $U \subset \mathcal{A}_{\left(c_{n}\right)}(\infty)$. Let $\varrho>0$ such that $D_{\varrho}(z) \subset U$. If $z^{\prime} \in D_{\varrho}(z)$ and $w^{\prime}:=F_{k}\left(z^{\prime}\right)$, then

$$
\begin{aligned}
w^{\prime}-w & =F_{k}\left(z^{\prime}\right)-F_{k}(z)=\int_{z}^{z^{\prime}} F_{k}^{\prime}(\zeta) d \zeta=F_{k}^{\prime}\left(F_{k}^{-1}(w)\right) \int_{z}^{z^{\prime}} \frac{F_{k}^{\prime}(\zeta)}{F_{k}^{\prime}\left(F_{k}^{-1}(w)\right)} d \zeta \\
& =F_{k}^{\prime}(z) \int_{z}^{z^{\prime}} \frac{\left(F_{k}^{-1}\right)^{\prime}(w)}{\left(F_{k}^{-1}\right)^{\prime}\left(F_{k}(\zeta)\right)} d \zeta,
\end{aligned}
$$


where we integrate over the line segment $\left[z, z^{\prime}\right]$. By Lemma 4.2 we obtain

$$
\left|\frac{\left(F_{k}^{-1}\right)^{\prime}(w)}{\left(F_{k}^{-1}\right)^{\prime}\left(F_{k}(\zeta)\right)}\right| \leq 1+\alpha e^{\alpha \varepsilon}\left|w-F_{k}(\zeta)\right| \leq 1+\alpha e^{\alpha \varepsilon} \varepsilon \leq 1+\alpha e^{\alpha}
$$

and thus

$$
\left|w^{\prime}-w\right| \leq\left|F_{k}^{\prime}(z)\right|\left|z^{\prime}-z\right|\left(1+\alpha e^{\alpha}\right) \leq\left|F_{k}^{\prime}(z)\right| \varrho\left(1+\alpha e^{\alpha}\right) .
$$

Setting

$$
\varrho:=\frac{\varepsilon}{\left|F_{k}^{\prime}(z)\right|\left(1+\alpha e^{\alpha}\right)}
$$

we obtain $D_{\varrho}(z) \subset U$ and thus

$$
d(z) \geq \frac{\varepsilon}{\left|F_{k}^{\prime}(z)\right|\left(1+\alpha e^{\alpha}\right)}=\frac{\alpha_{1}}{\left|F_{k}^{\prime}(z)\right|} \quad\left(z \in U_{k}\right) .
$$

In order to prove the John property, let $w_{0}:=\infty$ and $z_{0} \in \mathcal{A}_{\left(c_{n}\right)}(\infty)$. We may assume that $z_{0} \in U_{k} \backslash U_{k-1}$ for some $k \in \mathbb{N}$. Then $R<\left|F_{k}\left(z_{0}\right)\right| \leq R^{2}+\delta$. We construct an arc in $U_{k}$ joining $z_{0}$ and $w_{0}$ as follows. First, we join $z_{0}$ with $\partial U_{k-1}$ by an arc $\gamma_{k} \subset U_{k} \backslash U_{k-1}$ such that $F_{k}\left(\gamma_{k}\right) \subset \Gamma_{F_{k}\left(z_{0}\right)}$, and we denote the endpoint of $\gamma_{k}$ on $\partial U_{k-1}$ by $\zeta_{k-1}$. Then we join $\zeta_{k-1}$ with $\partial U_{k-2}$ by an arc $\gamma_{k-1} \subset U_{k-1} \backslash U_{k-2}$ such that $F_{k-1}\left(\gamma_{k-1}\right) \subset \Gamma_{F_{k-1}\left(\zeta_{k-1}\right)}$, and we denote the endpoint of $\gamma_{k-1}$ on $\partial U_{k-2}$ by $\zeta_{k-2}$. Proceeding in this way we get an arc in $U_{k} \cap \bar{D}_{R}$ with endpoint $\zeta_{0}$ on $\partial D_{R}$. Finally, we set $\gamma=\gamma\left(z_{0}\right):=\gamma_{k} \cup \cdots \cup \gamma_{1} \cup \Gamma_{\zeta_{0}}$. We note that the line segments $F_{j}\left(\gamma_{j}\right)$ $(j=1, \ldots, k)$ all lie in $\bar{\Delta}_{R} \cap \bar{D}_{R^{2}+\delta}$ and thus have lengths at most $\frac{1}{2}$.

We now show that the arc $\gamma$ has the John property. For that purpose, let $z \in \gamma$. We may assume that $z \in D_{R}$. First, let $z \in U_{k} \backslash U_{k-1}$. We deduce an upper estimate for $\left|z-z_{0}\right|$. There holds

$$
\begin{aligned}
z-z_{0} & =F_{k}^{-1}\left(F_{k}(z)\right)-F_{k}^{-1}\left(F_{k}\left(z_{0}\right)\right)=\int_{F_{k}\left(z_{0}\right)}^{F_{k}(z)}\left(F_{k}^{-1}\right)^{\prime}(\zeta) d \zeta \\
& =\left(F_{k}^{-1}\right)^{\prime}\left(F_{k}(z)\right) \int_{F_{k}\left(z_{0}\right)}^{F_{k}(z)} \frac{\left(F_{k}^{-1}\right)^{\prime}(\zeta)}{\left(F_{k}^{-1}\right)^{\prime}\left(F_{k}(z)\right)} d \zeta,
\end{aligned}
$$

where we integrate over the line segment $\left[F_{k}\left(z_{0}\right), F_{k}(z)\right]$. By Lemma 4.2 we obtain $\left|\frac{\left(F_{k}^{-1}\right)^{\prime}(\zeta)}{\left(F_{k}^{-1}\right)^{\prime}\left(F_{k}(z)\right)}\right| \leq 1+\alpha e^{\alpha}\left|F_{k}(z)-\zeta\right| \leq 1+\alpha e^{\alpha}\left|F_{k}(z)-F_{k}\left(z_{0}\right)\right| \leq 1+\alpha e^{\alpha}$ and thus

$$
\left|z-z_{0}\right| \leq\left|\left(F_{k}^{-1}\right)^{\prime}\left(F_{k}(z)\right)\right|\left(1+\alpha e^{\alpha}\right)\left|F_{k}(z)-F_{k}\left(z_{0}\right)\right|
$$




$$
\leq \frac{1+\alpha e^{\alpha}}{\left|F_{k}^{\prime}(z)\right|}=\frac{\alpha_{2}}{\left|F_{k}^{\prime}(z)\right|} \quad\left(z \in \gamma \backslash U_{k-1}\right) .
$$

Putting (4.2) and (4.3) together we arrive at

$$
d(z) \geq \frac{\alpha_{1}}{\alpha_{2}}\left|z-z_{0}\right|=\alpha_{3}\left|z-z_{0}\right| \quad\left(z \in \gamma \backslash U_{k-1}\right) .
$$

Now, let $z \in U_{k-m} \backslash U_{k-m-1}$ for some $m \in\{1, \ldots, k-1\}$. By (4.2) we have

$$
d(z) \geq \frac{\alpha_{1}}{\left|F_{k-m}^{\prime}(z)\right|} .
$$

From the construction of $\gamma$ and (4.3) we obtain

$$
\begin{gathered}
\left|z-z_{0}\right| \leq\left|z_{0}-\zeta_{k-1}\right|+\left|\zeta_{k-1}-\zeta_{k-2}\right|+\cdots+\left|\zeta_{k-m+1}-\zeta_{k-m}\right|+\left|\zeta_{k-m}-z\right| \\
\leq \alpha_{2}\left(\frac{1}{\left|F_{k}^{\prime}\left(\zeta_{k-1}\right)\right|}+\frac{1}{\left|F_{k-1}^{\prime}\left(\zeta_{k-2}\right)\right|}+\cdots\right. \\
\left.\quad+\frac{1}{\left|F_{k-m+1}^{\prime}\left(\zeta_{k-m}\right)\right|}+\frac{1}{\left|F_{k-m}^{\prime}(z)\right|}\right)
\end{gathered}
$$

and thus

$$
\frac{d(z)}{\left|z-z_{0}\right|} \geq \frac{\alpha_{3}}{1+\sum_{j=1}^{m}\left|\frac{F_{k-m}^{\prime}(z)}{F_{k-m+j}^{\prime}\left(\zeta_{k-m+j-1}\right)}\right|}
$$

In order to estimate the denominator of the right hand side we consider a single term

$$
\begin{aligned}
\frac{F_{k-m}^{\prime}(z)}{F_{k-m+j}^{\prime}\left(\zeta_{k-m+j-1}\right)}= & \frac{1}{2^{j} F_{k-m+j-1}\left(\zeta_{k-m+j-1}\right) \cdots F_{k-m}\left(\zeta_{k-m+j-1}\right)} \\
& \times \frac{F_{k-m}^{\prime}(z)}{F_{k-m}^{\prime}\left(\zeta_{k-m+j-1}\right)} .
\end{aligned}
$$

Because of $\left|F_{k-m+j-1}\left(\zeta_{k-m+j-1}\right)\right|=R$ and the invariance of $D_{r}$ we obtain

$$
\left|\frac{F_{k-m}^{\prime}(z)}{F_{k-m+j}^{\prime}\left(\zeta_{k-m+j-1}\right)}\right| \leq q^{j}\left|\frac{F_{k-m}^{\prime}(z)}{F_{k-m}^{\prime}\left(\zeta_{k-m+j-1}\right)}\right|,
$$

where $q:=\frac{1}{2 r}<1$.

Now, we deduce an estimate of the right hand side of (4.5). For abbreviation we set $p:=k-m$ and write

$$
\frac{F_{p}^{\prime}(z)}{F_{p}^{\prime}\left(\zeta_{p+j-1}\right)}=\frac{\left(F_{p}^{-1}\right)^{\prime}\left(F_{p}\left(\zeta_{p+j-1}\right)\right)}{\left(F_{p}^{-1}\right)^{\prime}\left(F_{p}(z)\right)} .
$$


From Lemma 4.2 we get

$$
\left|\frac{F_{p}^{\prime}(z)}{F_{p}^{\prime}\left(\zeta_{p+j-1}\right)}\right| \leq 1+\alpha \ell(\sigma) e^{\alpha \ell(\sigma)},
$$

where $\sigma=\sigma_{p, j}$ is the curve $F_{p}\left(\gamma_{p}^{\prime} \cup \gamma_{p+1} \cup \cdots \cup \gamma_{p+j-1}\right)$, and where $\gamma_{p}^{\prime}$ is that part of $\gamma_{p}$ joining $\zeta_{p}$ with $z$. Hence, there holds $\ell(\sigma) \leq \ell\left(F_{p}\left(\gamma_{p}\right)\right)+\cdots+$ $\ell\left(F_{p}\left(\gamma_{p+j-1}\right)\right)$. We have $\ell\left(F_{p}\left(\gamma_{p}\right)\right) \leq \frac{1}{2}$ and $F_{p}\left(\gamma_{p+\nu}\right)=F_{p+\nu, p}^{-1}\left(s_{p, \nu}\right)$, where $s_{p, \nu}:=F_{p+\nu}\left(\gamma_{p+\nu}\right)$ is a line segment on $\Gamma_{F_{p+\nu}\left(\zeta_{p+\nu}\right)}$ of length at most $\frac{1}{2}$ for $\nu \geq 1$. Furthermore, we know that $F_{p}\left(\gamma_{p+\nu}\right) \subset \Delta_{r}$. Therefore, we obtain

$$
\ell\left(F_{p}\left(\gamma_{p+1}\right)\right)=\int_{s_{p, 1}} \frac{|d w|}{2 \sqrt{\left|w-c_{p+1}\right|}} \leq \frac{\ell\left(s_{p, 1}\right)}{2 r} \leq \frac{1}{4 r} .
$$

By induction we get $\ell\left(F_{p}\left(\gamma_{p+\nu}\right)\right) \leq \frac{1}{2(2 r)^{\nu}}=\frac{1}{2} q^{\nu}$ and thus

$$
\ell(\sigma) \leq \frac{1}{2}\left(1+q+\cdots+q^{j-1}\right) \leq \frac{1}{2(1-q)}=\alpha_{4} .
$$

Setting $\alpha_{5}:=1+\alpha \alpha_{4} e^{\alpha \alpha_{4}}$ we obtain together with (4.4), (4.5) and (4.6)

$$
\frac{d(z)}{\left|z-z_{0}\right|} \geq \frac{\alpha_{3}}{1+\alpha_{5} \sum_{j=1}^{m} q^{j}} \geq \frac{\alpha_{3}(1-q)}{\alpha_{5}}
$$

which finally shows that $\gamma$ has the John property.

Theorem 4.4. Let $\delta<\frac{1}{4}$ and $\left(c_{n}\right) \in K_{\delta}^{\mathbb{N}}$. Then $\mathcal{A}_{\left(c_{n}\right)}(0)$ is a John domain.

Proof. The proof is very similar to the proof of Theorem 4.3. The only difficulty that arises is that $\mathcal{A}_{\left(c_{n}\right)}(0)$ contains critical values which all lie in $D_{s_{\delta}}$. Therefore, we only give a sketch and omit the details.

Let $\frac{1}{2}<r<r^{\prime}<r_{\delta}, \varepsilon:=r^{\prime}-r \leq 1$ and $U_{k}:=F_{k}^{-1}\left(D_{r^{\prime}}\right)$ for $k \in \mathbb{N}$. Then we have $U_{k} \subset U_{k+1} \subset \mathcal{A}_{\left(c_{n}\right)}(0)$ and $\mathcal{A}_{\left(c_{n}\right)}(0)=\bigcup_{k=1}^{\infty} U_{k}$. For $z \in \mathcal{A}_{\left(c_{n}\right)}(0)$ let $d(z):=\operatorname{dist}\left(z, \mathcal{J}_{\left(c_{n}\right)}\right)$. If $z \in U_{k} \backslash U_{k-1}$ for some $k \in \mathbb{N}, k \geq 2$ and $w:=F_{k-1}(z)$, then $|w| \geq r^{\prime}$ and thus $D_{\varepsilon}(w) \cap D_{r}=\varnothing$. Therefore, we obtain

$$
d(z) \geq \frac{\alpha_{1}}{\left|F_{k-1}^{\prime}(z)\right|} \quad\left(z \in U_{k} \backslash U_{k-1}\right) .
$$

In order to prove the John property, let $w_{0}:=0$ and $z_{0} \in \mathcal{A}_{\left(c_{n}\right)}(0)$. We may assume that $z_{0} \in U_{k} \backslash U_{k-1}$ for some $k \in \mathbb{N}, k \geq 2$. Then $\left|F_{k-1}\left(z_{0}\right)\right| \geq r^{\prime}$. We construct an arc in $U_{k}$ joining $z_{0}$ and $w_{0}$ as follows. First, we join $z_{0}$ with $\partial U_{k-1}$ by an arc $\gamma_{k} \subset U_{k} \backslash U_{k-1}$ such that $F_{k-1}\left(\gamma_{k}\right) \subset\left[0, F_{k-1}\left(z_{0}\right)\right]$, and we denote the endpoint of $\gamma_{k}$ on $\partial U_{k-1}$ by $\zeta_{k-1}$. Then we join $\zeta_{k-1}$ with $\partial U_{k-2}$ by an arc $\gamma_{k-1} \subset U_{k-1} \backslash U_{k-2}$ such that $F_{k-2}\left(\gamma_{k-1}\right) \subset\left[0, F_{k-2}\left(\zeta_{k-1}\right)\right]$, and we denote the endpoint of $\gamma_{k-1}$ on $\partial U_{k-2}$ by $\zeta_{k-2}$. Proceeding in this way we get an arc in $U_{k} \cap\left(\mathbb{C} \backslash U_{1}\right)$ with endpoint $\zeta_{1}$ on $\partial U_{1}$. Finally, we set $\gamma=\gamma\left(z_{0}\right):=\gamma_{k} \cup \cdots \cup \gamma_{2} \cup\left[0, \zeta_{1}\right]$. We mention that $\left[0, \zeta_{1}\right] \subset \bar{U}_{1}$, since $U_{1}$ 
is a starlike domain with respect to 0 bounded by an analytic Jordan curve. Furthermore, we note that the line segments $F_{j-1}\left(\gamma_{j}\right)(j=2, \ldots, k)$ all lie in $\bar{\Delta}_{r^{\prime}} \cap \bar{D}_{R_{\delta}}$ and thus have lengths at most one.

We now show that the arc $\gamma$ has the John property. For that purpose, let $z \in \gamma$. We may assume that $z \notin U_{1}$. First, let $z \in U_{k} \backslash U_{k-1}$. Then we obtain the upper estimate for $\left|z-z_{0}\right|$

$$
\left|z-z_{0}\right| \leq \frac{\alpha_{2}}{\left|F_{k-1}^{\prime}(z)\right|} \quad\left(z \in \gamma \backslash U_{k-1}\right) .
$$

Putting (4.2a) and (4.3a) together we arrive at

$$
d(z) \geq \alpha_{3}\left|z-z_{0}\right| \quad\left(z \in \gamma \backslash U_{k-1}\right) .
$$

Finally, the case that $z \in U_{k-m} \backslash U_{k-m-1}$ for some $m \in\{1, \ldots, k-2\}$ is handled as in the proof of Theorem 4.3.

Corollary 4.5. Let $\delta<\frac{1}{4}$ and $\left(c_{n}\right) \in K_{\delta}^{\mathbb{N}}$. Then $\mathcal{J}_{\left(c_{n}\right)}$ is a quasicircle.

Proof. From Theorem 4.1 we know that $\mathcal{F}_{\left(c_{n}\right)}=\mathcal{A}_{\left(c_{n}\right)}(\infty) \cup \mathcal{A}_{\left(c_{n}\right)}(0)$. Then the assertion follows from Theorems 4.3 and 4.4 and the known results mentioned at the beginning of this section.

If $\left(c_{n}\right) \in K_{1 / 4}^{\mathbb{N}}$, then $\mathcal{J}_{\left(c_{n}\right)}$ need not be a quasicircle. For example, if $c_{n}=\frac{1}{4}$ for all $n$, then it is known that $\mathcal{J}\left(f_{1 / 4}\right)$ is still a Jordan curve (see for example [CG, p. 97] or [St, p. 124]) but it has cusps. Furthermore, Corollary 4.5 does not hold true in general when all $c_{n}$ are contained in the interior of the main cardioid of the Mandelbrot set. This can be seen by the simple example $c_{1}=-\frac{1}{2}-\eta$ and $c_{n}=\frac{1}{4}-\varepsilon$ for $n \geq 2$ with $0<\eta<\frac{1}{4}$ and $0<\varepsilon<\eta^{2}$. In this case we have $F_{n}(0) \rightarrow \infty$ as $n \rightarrow \infty$ so that by Theorem 1.1 in [BBR] the Julia set $\mathcal{J}_{\left(c_{n}\right)}$ is even disconnected. It would be of interest whether $\mathcal{J}_{\left(c_{n}\right)}$ is also a Jordan curve in our more general setting provided that $\left(c_{n}\right) \in K_{1 / 4}^{\mathbb{N}}$ or what holds when $\left(c_{n}\right) \in D_{1 / 4}^{\mathbb{N}}$.

Furthermore, we consider the dynamics of $\left(F_{n}\right)$ in the stable domain $\mathcal{A}_{\left(c_{n}\right)}(0)$ provided that $\left(c_{n}\right) \in K_{1 / 4}^{\mathbb{N}}$. We will show that $\mathcal{A}_{\left(c_{n}\right)}(0)$ is a contracting domain, that is a stable domain $U$ such that all limit functions of $\left(F_{n}\right)$ in $U$ are constant. This property is equivalent to diam $F_{n}(K) \rightarrow 0$ as $n \rightarrow \infty$ for every compact set $K \subset U$.

Theorem 4.6. Let $\left(c_{n}\right) \in K_{1 / 4}^{\mathbb{N}}$. Then $\mathcal{A}_{\left(c_{n}\right)}(0)$ is a contracting domain.

Proof. Let $K \subset \mathcal{A}_{\left(c_{n}\right)}(0)$ be a compact set. We first assume that $\left(c_{n}\right) \in K_{\delta}^{\mathbb{N}}$ for some $\delta<\frac{1}{4}$, and we choose $r \in\left(s_{\delta}, \frac{1}{2}\right)$. Then by Theorem 4.1 there exists $N \in \mathbb{N}$ such that $F_{N}(K) \subset D_{r}$. If $z_{1}, z_{2} \in K$, then $w_{1}:=F_{N}\left(z_{1}\right)$, $w_{2}:=F_{N}\left(z_{2}\right) \in D_{r}$ and thus $\left|f_{c_{k}}\left(w_{1}\right)-f_{c_{k}}\left(w_{2}\right)\right|=\left|w_{1}+w_{2}\right|\left|w_{1}-w_{2}\right| \leq$ $2 r\left|w_{1}-w_{2}\right|$ which implies $\left|F_{N+k}\left(z_{1}\right)-F_{N+k}\left(z_{2}\right)\right| \leq(2 r)^{k}\left|w_{1}-w_{2}\right|$. Therefore, 
we obtain $\operatorname{diam} F_{N+k}(K) \leq(2 r)^{k} \operatorname{diam} F_{N}(K) \rightarrow 0$ as $k \rightarrow \infty$, and the assertion follows.

Now, let $\left|c_{n}\right| \leq \frac{1}{4}$ for all $n \in \mathbb{N}$. Again, by Theorem 4.1 there exists $N \in \mathbb{N}$ such that $F_{N}(K) \subset K_{1 / 2}$, and we obtain as above $\operatorname{diam} F_{N+k}(K) \leq$ $\operatorname{diam} F_{N+k-1}(K)$ so that the sequence (diam $F_{N+k}(K)$ ) is monotonically decreasing and thus convergent. In order to deduce diam $F_{N+k}(K) \rightarrow 0$ as $k \rightarrow \infty$ we need a better estimate. If $w_{1}, w_{2} \in F_{N}(K)$, we obtain

$$
\left|f_{c_{k}}\left(w_{1}\right)-f_{c_{k}}\left(w_{2}\right)\right| \leq 2\left|\int_{w_{1}}^{w_{2}}\right| z|| d z|| .
$$

For the estimate of the right hand side we consider the worst case which can happen, that is $\left|w_{1}\right|=\left|w_{2}\right|=\frac{1}{2}$. For simplicity, we may assume that $w_{2}=\bar{w}_{1}$, and we set $\varrho:=\operatorname{Re} w_{1}=\operatorname{Re} w_{2} \in\left[0, \frac{1}{2}\right)$. Then with $d:=\frac{1}{2}\left|w_{1}-w_{2}\right|$ we get $\varrho^{2}+d^{2}=\frac{1}{4}$ and thus

$$
\begin{aligned}
2\left|\int_{w_{1}}^{w_{2}}\right| z|| d z|| & \leq 4 \int_{0}^{d}|\varrho+i t| d t=4 \int_{0}^{d} \sqrt{\varrho^{2}+t^{2}} d t \\
& =d+2 \varrho^{2} \log \frac{2 d+1}{2 \varrho} \\
& =\frac{1}{2}\left|w_{1}-w_{2}\right|+\frac{1}{4}\left(1-\left|w_{1}-w_{2}\right|^{2}\right) \log \frac{1+\left|w_{1}-w_{2}\right|}{1-\left|w_{1}-w_{2}\right|} .
\end{aligned}
$$

This implies with $d_{n}:=\operatorname{diam} F_{n}(K)$

$$
d_{N+k} \leq \frac{1}{2} d_{N+k-1}+\frac{1}{4}\left(1-d_{N+k-1}^{2}\right) \log \frac{1+d_{N+k-1}}{1-d_{N+k-1}} .
$$

Setting $\alpha:=\lim _{k \rightarrow \infty} \operatorname{diam} F_{N+k}(K)$ we see that

$$
\alpha \leq \frac{1}{2} \alpha+\frac{1}{4}\left(1-\alpha^{2}\right) \log \frac{1+\alpha}{1-\alpha},
$$

and an elementary argument shows that this is possible only for $\alpha=0$ which gives the assertion.

If $\left(c_{n}\right) \in K_{\delta}^{\mathbb{N}}$ for some $\delta \leq \frac{1}{4}$, we denote by $L_{\left(c_{n}\right)}$ the set of (constant) limit functions of $\left(F_{n}\right)$ in $\mathcal{A}_{\left(c_{n}\right)}(0)$, that is the set of all $\zeta \in \mathbb{C}$ such that for some subsequence $\left(F_{n_{k}}\right)$ of $\left(F_{n}\right)$ there holds $F_{n_{k}} \rightarrow \zeta$ as $k \rightarrow \infty$ locally uniformly in $\mathcal{A}_{\left(c_{n}\right)}(0)$. It is easy to see that $L_{\left(c_{n}\right)}$ is a compact set, and from the proof of Theorem 4.6 it follows that $L_{\left(c_{n}\right)} \subset K_{s_{\delta}} \subset K_{1 / 2}$. From Theorem 1.6 in $[\mathbf{B B R}]$ we know that the case $L_{\left(c_{n}\right)}=K_{s_{\delta}}$ may occur. Moreover, this phenomenon happens almost surely, that means that the product measure (cf. Section 5) of the set of these sequences $\left(c_{n}\right)$ in $K_{\delta}^{\mathbb{N}}$ is one. In a similar way it is possible to construct sequences $\left(c_{n}\right) \in K_{\delta}^{\mathbb{N}}$ such that $L_{\left(c_{n}\right)}=\partial K_{s_{\delta}}$. 
On the other hand, if $L_{\left(c_{n}\right)}$ consists of a single point $\zeta$, then $F_{n} \rightarrow \zeta$ as $n \rightarrow \infty$ locally uniformly in $\mathcal{A}_{\left(c_{n}\right)}(0)$, and since $F_{n+1}(z)=\left(F_{n}(z)\right)^{2}+c_{n}$ we obtain $c_{n} \rightarrow c \in K_{\delta}$ as $n \rightarrow \infty$, where $c=\zeta-\zeta^{2}$. Therefore, the set $C_{\delta}$ of all these points $\zeta$ is the component of the preimage of $K_{\delta}$ under the map $z \mapsto z-z^{2}$ which is contained in $K_{s_{\delta}}$. Therefore, $C_{\delta}$ is a proper subset of $K_{s_{\delta}}$ and $C_{\delta} \cap \partial K_{s_{\delta}}=\left\{s_{\delta}\right\}$. It would be of interest to characterize those compact sets $K \subset K_{s_{\delta}}$ such that $K=L_{\left(c_{n}\right)}$ for some sequence $\left(c_{n}\right) \in K_{\delta}^{\mathbb{N}}$.

The stable domain $\mathcal{A}_{\left(c_{n}\right)}(\infty)$ may be viewed as a Böttcher domain. If it is simply connected, then there exists a conformal map $\phi$ of $\mathcal{A}_{\left(c_{n}\right)}(\infty)$ onto $\Delta_{1}$ normalized at infinity by

$$
\phi(z)=z+a_{0}+\frac{a_{1}}{z}+\frac{a_{2}}{z^{2}}+\cdots .
$$

Note that the capacity of $\mathcal{K}_{\left(c_{n}\right)}$ (cf. Section 8) is equal to one. Like in the iteration of a fixed polynomial we show that $\phi$ may be described dynamically.

Theorem 4.7. Let $\delta>0$ and $\left(c_{n}\right) \in K_{\delta}^{\mathbb{N}}$ such that $\mathcal{A}_{\left(c_{n}\right)}(\infty)$ is simply connected. Then the conformal map $\phi$ of $\mathcal{A}_{\left(c_{n}\right)}(\infty)$ onto $\Delta_{1}$ with the normalization (4.7) is given by

$$
\phi(z)=\lim _{k \rightarrow \infty} \sqrt[2^{k}]{F_{k}(z)}=z \lim _{k \rightarrow \infty} \sqrt[2^{k}]{\frac{F_{k}(z)}{z^{2^{k}}}}
$$

with locally uniform convergence in $\mathcal{A}_{\left(c_{n}\right)}(\infty)$, and where the branch of the root is determined by $\sqrt[2^{k}]{1}=1$.

Proof. Let $R>R_{\delta}$ such that $R^{2} \geq 2 \delta$ and $U_{m}:=F_{m}^{-1}\left(\Delta_{R}\right)$ for $m \in \mathbb{N}$. Then we have $U_{m} \subset U_{m+1} \subset \mathcal{A}_{\left(c_{n}\right)}(\infty)$ and $\mathcal{A}_{\left(c_{n}\right)}(\infty)=\bigcup_{m=1}^{\infty} U_{m}$. For $k \in \mathbb{N}$ we define

$$
\phi_{k}(z):=\sqrt[2^{k}]{F_{k}(z)}=z \sqrt[2^{k}]{\frac{F_{k}(z)}{z^{2^{k}}}}
$$

Then $\phi_{k}$ maps $U_{k}$ conformally onto $\Delta_{R_{k}}$, where $R_{k}:=\sqrt[2^{k}]{R}$. For $z \in U_{m}$ and $k \geq m$ we have

$$
\left|\frac{c_{k}}{\left(F_{k}(z)\right)^{2}}\right| \leq \frac{\delta}{R^{2}} \leq \frac{1}{2}
$$

and the elementary inequality

$$
|\sqrt[p]{1+u}-1| \leq \frac{1}{p} \quad\left(u \in K_{1 / 2}\right)
$$

yields

$$
\left|\frac{\phi_{k+1}(z)}{\phi_{k}(z)}-1\right|=\left|\sqrt[2^{k+1}]{\frac{F_{k+1}(z)}{\left(F_{k}(z)\right)^{2}}}-1\right|=\left|2^{k+1} \sqrt{1+\frac{c_{k}}{\left(F_{k}(z)\right)^{2}}}-1\right| \leq \frac{1}{2^{k+1}} .
$$


Therefore, the limit

$$
\phi(z):=\lim _{k \rightarrow \infty} \phi_{k}(z)=z \prod_{k=0}^{\infty} \frac{\phi_{k+1}(z)}{\phi_{k}(z)}
$$

exists uniformly in $U_{m}$, and $\phi$ is the desired conformal map.

\section{Lebesgue measure of Julia sets.}

From a result of Lyubich [L2] (see also [CG, p. 90] or [St, p. 144]) it follows that the Julia set of a hyperbolic rational function has two-dimensional Lebesgue measure (which we denote by $m_{2}$ ) zero. In particular, this is true for $\mathcal{J}\left(f_{c}\right)$ provided that $c$ is contained in a hyperbolic component of the interior of the Mandelbrot set $\mathcal{M}$ or $c \notin \mathcal{M}$. In this section we show that this is true to a certain extent in our situation.

We begin with $\delta<\frac{1}{4}$. Then by Section 4 we know that if $\left(c_{n}\right) \in K_{\delta}^{\mathbb{N}}$, then $\mathcal{J}_{\left(c_{n}\right)}$ is a quasicircle, and from the differentiability properties of quasiconformal maps it follows that quasicircles always have two-dimensional Lebesgue measure zero (see for example $[\mathbf{L V}$, p. 165]).

Corollary 5.1. Let $\delta<\frac{1}{4}$ and $\left(c_{n}\right) \in K_{\delta}^{\mathbb{N}}$. Then $m_{2}\left(\mathcal{J}_{\left(c_{n}\right)}\right)=0$.

Now, we will show that $m_{2}\left(\mathcal{J}_{\left(c_{n}\right)}\right)$ is almost surely zero provided that the $c_{n}$ are randomly chosen in $K_{\delta}$ for some $\delta>\frac{1}{4}$. To be more precise, let $\lambda_{\delta}$ denote the two-dimensional Lebesgue measure on $K_{\delta}$ normalized by $\lambda_{\delta}\left(K_{\delta}\right)=1$. Then the product space $K_{\delta}^{\mathbb{N}}$ carries the usual product measure $\tilde{\lambda}_{\delta}:=\bigotimes_{k=1}^{\infty} \lambda_{\delta}$. We set

$$
\mathfrak{N}_{\delta}:=\left\{\left(c_{n}\right) \in K_{\delta}^{\mathbb{N}}: m_{2}\left(\mathcal{J}_{\left(c_{n}\right)}\right)=0\right\} .
$$

Then the goal is to show that $\tilde{\lambda}_{\delta}\left(\mathfrak{N}_{\delta}\right)=1$. In order to do this we recall:

Theorem $5.2([\mathrm{BBR}])$. Let $\delta>\frac{1}{4}$ and $R>0$. Then for every $z \in \widehat{\mathbb{C}}$ there exists an open set $\mathfrak{U}_{z} \subset K_{\delta}^{\mathbb{N}}$ with the following properties:

(a) $\tilde{\lambda}_{\delta}\left(\mathfrak{U}_{z}\right)=1$

(b) for every $\left(c_{n}\right) \in \mathfrak{U}_{z}$ there holds $\left|F_{k}(z)\right|>R$ for all sufficiently large $k$.

Theorem 5.3. Let $\delta>\frac{1}{4}$, and let $\mathfrak{N}_{\delta} \subset K_{\delta}^{\mathbb{N}}$ be defined by (5.1). Then $\tilde{\lambda}_{\delta}\left(\mathfrak{N}_{\delta}\right)=1$.

Proof. Let $M=\Delta_{R}$ be an invariant domain and

$$
\widetilde{\mathfrak{E}}:=\left\{\left(\left(c_{n}\right), z\right) \in K_{\delta}^{\mathbb{N}} \times \widehat{\mathbb{C}}: F_{k}(z) \in M \text { for some } k \in \mathbb{N}\right\} .
$$

By Theorem 5.2 we have $\tilde{\lambda}_{\delta}\left(\widetilde{\mathfrak{E}}_{z}\right)=1$ for $z \in \widehat{\mathbb{C}}$, where

$$
\widetilde{\mathfrak{E}}_{z}:=\left\{\left(c_{n}\right) \in K_{\delta}^{\mathbb{N}}:\left(\left(c_{n}\right), z\right) \in \widetilde{\mathfrak{E}}\right\} .
$$


If $\sigma$ denotes the two-dimensional Lebesgue measure on $\widehat{\mathbb{C}}$ normalized by $\sigma(\widehat{\mathbb{C}})=1$, it follows

$$
\left(\tilde{\lambda}_{\delta} \otimes \sigma\right)(\widetilde{\mathfrak{E}})=\int_{\widehat{\mathbb{C}}} \tilde{\lambda}_{\delta}\left(\widetilde{\mathfrak{E}}_{z}\right) d \sigma(z)=1
$$

Now, let

$$
\mathfrak{E}:=\left\{\left(c_{n}\right) \in K_{\delta}^{\mathbb{N}}: \sigma\left(\widetilde{\mathfrak{E}}_{\left(c_{n}\right)}\right)=1\right\},
$$

where

$$
\widetilde{\mathfrak{E}}_{\left(c_{n}\right)}:=\left\{z \in \widehat{\mathbb{C}}:\left(\left(c_{n}\right), z\right) \in \widetilde{\mathfrak{E}}\right\} .
$$

Since

$$
1=\left(\tilde{\lambda}_{\delta} \otimes \sigma\right)(\widetilde{\mathfrak{E}})=\int_{K_{\delta}^{\mathbb{N}}} \sigma\left(\widetilde{\mathfrak{E}}_{\left(c_{n}\right)}\right) d \tilde{\lambda}_{\delta}\left(\left(c_{n}\right)\right)
$$

we obtain $\tilde{\lambda}_{\delta}(\mathfrak{E})=1$. If $\left(c_{n}\right) \in \mathfrak{E}$, then

$$
\sigma\left(\mathcal{A}_{\left(c_{n}\right)}(\infty)\right)=\sigma\left(\left\{z \in \widehat{\mathbb{C}}: F_{k}(z) \in M \text { for some } k \in \mathbb{N}\right\}\right)=\sigma\left(\widetilde{\mathfrak{E}}_{\left(c_{n}\right)}\right)=1
$$

which implies $\sigma\left(\mathcal{J}_{\left(c_{n}\right)}\right)=0$.

It would be of interest whether Theorem 5.3 remains valid for $\delta=\frac{1}{4}$. Concerning the question whether there exists a sequence $\left(c_{n}\right) \in K_{\delta}^{\mathbb{N}}$ for some $\delta \geq \frac{1}{4}$ such that $m_{2}\left(\mathcal{J}_{\left(c_{n}\right)}\right)>0$, the referee mentioned that, recently, a group of mathematicians around P.W. Jones at Yale University have constructed such an example. More precisely, there exists a sequence $\left(c_{n}\right)$ with $c_{n} \in$ $\left\{0, \pm \frac{1}{4}, \frac{1}{2}\right\}$ such that $m_{2}\left(\mathcal{J}_{\left(c_{n}\right)}\right)>0$. This result was communicated to the author by P.W. Jones. The author is grateful to both for bringing this information to his attention.

Finally, we prove:

Theorem 5.4. Let $\delta>2$ (which is equivalent to $\delta>R_{\delta}$ ), and let $\varepsilon>0$ such that $R_{\delta}+\varepsilon \leq\left|c_{n}\right| \leq \delta$ for all $n \in \mathbb{N}$. Then $m_{2}\left(\mathcal{J}_{\left(c_{n}\right)}\right)=0$.

Proof. We choose $R$ such that $R_{\delta}<R<R_{\delta}+\varepsilon$ and $\eta:=R_{\delta}+\varepsilon-R>0$. Then we have $\mathcal{J}_{\left(c_{n}\right)} \subset \bar{D}_{R_{\delta}} \subset D:=D_{R}$, and $D$ is backward invariant, that is $f_{c_{n}}^{-1}(D) \subset D$ for all $n \in \mathbb{N}$. Furthermore, there holds $\left|f_{c_{n}}(0)\right|=\left|c_{n}\right| \geq$ $R_{\delta}+\varepsilon=R+\eta$ and thus $\left|\left(f_{c_{m}} \circ \cdots \circ f_{c_{k+1}}\right)(0)\right| \geq R+\eta$ for $k=0,1, \ldots, m-1$ and all $m \in \mathbb{N}$. Therefore, $D$ does not contain any critical value of $\left(F_{n}\right)$ so that in $D$ there exist $2^{k}$ analytic branches of the inverse function of $F_{k}$ which we denote by $G_{j, k}$ for $j=1, \ldots, 2^{k}$ and $k \in \mathbb{N}$. Furthermore, we set $D_{j, k}:=G_{j, k}(D) \subset D$ and $D_{k}:=\bigcup_{j=1}^{2^{k}} D_{j, k}$. Then $D_{1, k}, \ldots, D_{2^{k}, k}$ are mutually disjoint simply connected domains, and $\mathcal{J}_{\left(c_{n}\right)} \subset D_{k+1} \subset D_{k}$. Finally, we set $U_{k}:=D_{k} \backslash \bar{D}_{k+1}$ and $U_{j, k}:=D_{j, k} \backslash \bar{D}_{k+1}$ so that $U_{1, k}, \ldots, U_{2^{k}, k}$ are mutually disjoint multiply connected domains, and $U_{k}=\bigcup_{j=1}^{2^{k}} U_{j, k}$. 
Now, we prove that there exists a constant $q>0$ such that

$$
\frac{m_{2}\left(U_{k}\right)}{m_{2}\left(D_{k}\right)} \geq q
$$

for all $k \in \mathbb{N}$. For that purpose it is enough to show that

$$
\frac{m_{2}\left(U_{j, k}\right)}{m_{2}\left(D_{j, k}\right)} \geq q
$$

for $j=1, \ldots, 2^{k}$ and all $k \in \mathbb{N}$.

Let $V_{1, k}$ and $V_{2, k}$ denote the two components of $f_{c_{k+1}}^{-1}(D)$, and let $W_{k}:=$ $D \backslash\left(\bar{V}_{1, k} \cup \bar{V}_{2, k}\right)$. Then $U_{j, k}=G_{j, k}\left(W_{k}\right)$, and we obtain

$$
\frac{m_{2}\left(U_{j, k}\right)}{m_{2}\left(D_{j, k}\right)}=\frac{\int_{W_{k}}\left|G_{j, k}^{\prime}(z)\right|^{2} d m_{2}(z)}{\int_{D}\left|G_{j, k}^{\prime}(z)\right|^{2} d m_{2}(z)} \geq \frac{\left|G_{j, k}^{\prime}\left(z_{j, k}\right)\right|^{2} m_{2}\left(W_{k}\right)}{\left|G_{j, k}^{\prime}\left(\zeta_{j, k}\right)\right|^{2} m_{2}(D)},
$$

where $z_{j, k} \in W_{k} \subset D$ and $\zeta_{j, k} \in D$ such that $\left|G_{j, k}^{\prime}\left(z_{j, k}\right)\right|=\min _{z \in W_{k}}\left|G_{j, k}^{\prime}(z)\right|$ and $\left|G_{j, k}^{\prime}\left(\zeta_{j, k}\right)\right|=\max _{z \in D}\left|G_{j, k}^{\prime}(z)\right|$. By the Koebe distortion theorem (see for example $\left[\mathbf{P 2}\right.$, p. 9]) applied to the disk $D_{R+\eta}$ there holds

$$
\left|\frac{G_{j, k}^{\prime}(z)}{G_{j, k}^{\prime}(\zeta)}\right| \geq\left(\frac{\eta}{\eta+2 R}\right)^{4}
$$

for all $z, \zeta \in D$. Therefore, it remains to show that there exists a constant $\gamma>0$ such that

$$
\frac{m_{2}\left(W_{k}\right)}{m_{2}(D)} \geq \gamma
$$

for all $k \in \mathbb{N}$.

For simplicity we write $c=c_{k+1}$, and let $V \in\left\{V_{1, k}, V_{2, k}\right\}$. Then

$$
m_{2}(V)=\frac{1}{4} \int_{D} \frac{d m_{2}(z)}{|z-c|}=\frac{1}{4} \int_{0}^{R} \int_{0}^{2 \pi} \frac{\varrho}{\left|\varrho e^{i t}-c\right|} d t d \varrho .
$$

By the Cauchy-Schwarz inequality we get

$$
\int_{0}^{2 \pi} \frac{d t}{\left|\varrho e^{i t}-c\right|} \leq \sqrt{2 \pi}\left(\int_{0}^{2 \pi} \frac{d t}{\left|\varrho e^{i t}-c\right|^{2}}\right)^{1 / 2}
$$

and the Poisson integral formula yields

$$
\int_{0}^{2 \pi} \frac{d t}{\left|\varrho e^{i t}-c\right|^{2}}=\frac{2 \pi}{|c|^{2}-\varrho^{2}} .
$$

Therefore, we arrive at

$$
m_{2}(V) \leq \frac{\pi}{2} \int_{0}^{R} \frac{\varrho}{\sqrt{|c|^{2}-\varrho^{2}}} d \varrho=\frac{\pi}{2}\left(|c|-\sqrt{|c|^{2}-R^{2}}\right) \leq \frac{1}{2} \pi R .
$$


This implies $m_{2}\left(V_{1, k} \cup V_{2, k}\right) \leq \pi R$ and thus

$$
\frac{m_{2}\left(W_{k}\right)}{m_{2}(D)} \geq 1-\frac{1}{R} \geq \frac{1}{2}
$$

which proves (5.3).

Finally, (5.2) gives $m_{2}\left(\mathcal{J}_{\left(c_{n}\right)}\right) \leq m_{2}\left(D_{k+1}\right)=m_{2}\left(D_{k}\right)-m_{2}\left(U_{k}\right) \leq(1-$ q) $m_{2}\left(D_{k}\right)$ so that $m_{2}\left(\mathcal{J}_{\left(c_{n}\right)}\right) \leq(1-q)^{k} m_{2}(D) \rightarrow 0$ as $k \rightarrow \infty$ which completes the proof.

\section{Hausdorff dimension of Julia sets.}

We first recall the notion of Hausdorff dimension. Let $E \subset \mathbb{C}$ be a nonempty compact set, and denote by $\left(D_{j}\right)_{\varepsilon}$ any covering of $E$ by finitely many open sets $D_{j}$ with diam $D_{j}<\varepsilon$. Then for $t \in(0,2]$

$$
m_{t}(E):=\sup _{\varepsilon>0} \inf _{\left(D_{j}\right)_{\varepsilon}} \sum_{j}\left(\operatorname{diam} D_{j}\right)^{t}
$$

is called the $t$-dimensional Hausdorff measure of $E$. Obviously, $m_{t}(E)<\infty$ implies $m_{s}(E)=0$ for $s>t$, and conversely, $m_{t}(E)>0$ implies $m_{s}(E)=\infty$ for $s<t$. Hence, there exists a unique $\tau \in[0,2]$ such that $m_{s}(E)=0$ and $m_{t}(E)=\infty$ for $0<t<\tau<s \leq 2$. This number $\tau$ is called the Hausdorff dimension of $E$ and is denoted by $\operatorname{dim}_{H} E$.

It is well-known (cf. [G], see also [Be, p. 251] or [St, p. 169]) that the Hausdorff dimension of the Julia set of any fixed rational function $f$ is positive. More precisely, if $\infty \notin \mathcal{J}(f)$ and if $d$ denotes the degree of $f$, then

$$
\operatorname{dim}_{\mathrm{H}} \mathcal{J}(f) \geq \frac{\log d}{\log \max _{z \in \mathcal{J}(f)}\left|f^{\prime}(z)\right|} .
$$

We show that this estimate holds true in a certain sense in our situation.

Theorem 6.1. Let $\delta>0$ and $\left(c_{n}\right) \in K_{\delta}^{\mathbb{N}}$. Then $\operatorname{dim}_{\mathrm{H}} \mathcal{\partial}_{\left(c_{n}\right)}>0$. More precisely, there holds

$$
\operatorname{dim}_{\mathrm{H}} \partial_{\left(c_{n}\right)} \geq \frac{\log 2}{\log \left(2 R_{\delta}\right)}=\frac{\log 2}{\log (1+\sqrt{1+4 \delta})} .
$$

Proof. We show that the Green function $g$ of $\mathcal{A}_{\left(c_{n}\right)}(\infty)$ is Hölder continuous with exponent

$$
\alpha=\frac{\log 2}{\log 2+\log \left(2 R-R_{\delta}\right)}
$$

for any $R>R_{\delta}$. Then a result of Carleson $[\mathbf{C}]$ gives $\operatorname{dim}_{\mathrm{H}} \mathcal{J}_{\left(c_{n}\right)} \geq \alpha$. For that purpose, it suffices to show that there exists a constant $\gamma>0$ such that $g(z) \leq \gamma(d(z))^{\alpha}$ for all $z \in \mathcal{A}_{\left(c_{n}\right)}(\infty)$, where $d(z):=\operatorname{dist}\left(z, \mathcal{J}_{\left(c_{n}\right)}\right)$. Of course, we may assume that $d(z)$ is small. 
Let $R>R_{\delta}$ and $U_{k}:=F_{k}^{-1}\left(\Delta_{R}\right)$ for $k \in \mathbb{N}$. Then we have $U_{k} \subset U_{k+1} \subset$ $\mathcal{A}_{\left(c_{n}\right)}(\infty)$ and $\mathcal{A}_{\left(c_{n}\right)}(\infty)=\bigcup_{k=1}^{\infty} U_{k}$. The Green function $g_{k}$ of $U_{k}$ with pole at infinity is given by

$$
g_{k}(z)=\frac{1}{2^{k}} \log \frac{\left|F_{k}(z)\right|}{R} \quad\left(z \in U_{k}\right) .
$$

There holds $g_{k}(z) \leq g_{k+1}(z) \leq g(z)$ for $z \in U_{k}$ and $g_{k} \rightarrow g$ as $k \rightarrow \infty$ locally uniformly in $\mathcal{A}_{\left(c_{n}\right)}(\infty)$.

We will show that there exists some constant $C>0$ such that $g(z) \leq$ $g_{k}(z)+\frac{C}{2^{k}}$ for $z \in U_{k}$. There holds $\left|F_{k+1}(z)\right|=\left|\left(F_{k}(z)\right)^{2}+c_{k+1}\right| \leq\left|F_{k}(z)\right|^{2}+\delta$ and this gives

$$
g_{k+1}(z) \leq \frac{1}{2^{k+1}} \log \frac{\left|F_{k}(z)\right|^{2}+\delta}{R} .
$$

If $a, b>0$, then $\log ^{+}(a+b) \leq \log ^{+} a+\log ^{+} b+\log 2$, and thus

$$
\begin{aligned}
g_{k+1}(z) & \leq \frac{1}{2^{k+1}}\left(\log \frac{\left|F_{k}(z)\right|^{2}}{R}+\log ^{+} \frac{\delta}{R}+\log 2\right) \\
& =\frac{1}{2^{k+1}}\left(2 \log \frac{\left|F_{k}(z)\right|}{R}+\log ^{+} \frac{\delta}{R}+\log (2 R)\right) \\
& =g_{k}(z)+\frac{C}{2^{k+1}},
\end{aligned}
$$

where $C:=\log ^{+} \frac{\delta}{R}+\log (2 R)$. From this we obtain by induction

$$
g_{k+m}(z) \leq g_{k}(z)+C\left(\frac{1}{2^{k+1}}+\cdots+\frac{1}{2^{k+m}}\right) \leq g_{k}(z)+\frac{C}{2^{k}}
$$

for all $m \in \mathbb{N}$. Letting $m \rightarrow \infty$ we get

$$
g(z) \leq g_{k}(z)+\frac{C}{2^{k}} \quad\left(z \in U_{k}\right) .
$$

Now, let $z \in U_{k} \backslash U_{k-1}$ for some $k \in \mathbb{N}$. Then $\left|F_{k-1}(z)\right| \leq R$ which implies $\left|F_{k}(z)\right| \leq R^{2}+\delta$. Hence, we have $g_{k}(z) \leq \frac{1}{2^{k}} \log \left(R+\frac{\delta}{R}\right)$ and thus

$$
g(z) \leq \frac{\Gamma}{2^{k}} \quad\left(z \in U_{k} \backslash U_{k-1}\right),
$$

where $\Gamma:=C+\log \left(R+\frac{\delta}{R}\right)$.

Finally, we prove a lower estimate for $d(z)$, if $z \in U_{k}$ for some $k \in \mathbb{N}$. We set $w:=F_{k}(z)$ and $\eta:=|w|-R_{\delta}$. If $U$ denotes the component of $F_{k}^{-1}\left(D_{\eta}(w)\right)$ containing $z$, there holds $U \subset \mathcal{A}_{\left(c_{n}\right)}(\infty)$. Let $\varrho>0$ such that $D_{\varrho}(z) \subset U$. Then $F_{k}\left(D_{\varrho}(z)\right) \subset D_{\eta}(w) \subset D_{|w|+\eta}$ which implies $F_{j}\left(D_{\varrho}(z)\right) \subset D_{|w|+\eta}$ for 
$j=0,1, \ldots, k$ and thus $\left|F_{k}^{\prime}(t)\right| \leq 2^{k}(|w|+\eta)^{k}$ for all $t \in D_{\varrho}(z)$. If $z^{\prime} \in D_{\varrho}(z)$ and $w^{\prime}:=F_{k}\left(z^{\prime}\right)$, then

$$
w^{\prime}-w=\int_{z}^{z^{\prime}} F_{k}^{\prime}(t) d t
$$

where we integrate over the line segment joining $z$ and $z^{\prime}$. This yields

$$
\left|w^{\prime}-w\right| \leq 2^{k}(|w|+\eta)^{k}\left|z^{\prime}-z\right|<2^{k}(|w|+\eta)^{k} \varrho .
$$

Setting

$$
\varrho:=\frac{\eta}{2^{k}(|w|+\eta)}
$$

we obtain $D_{\varrho}(z) \subset U$ and thus

$$
d(z) \geq \frac{\eta}{2^{k}(|w|+\eta)}=\frac{|w|-R_{\delta}}{2^{k}\left(2|w|-R_{\delta}\right)} \geq \frac{R-R_{\delta}}{2^{k}\left(2 R-R_{\delta}\right)} \quad\left(z \in U_{k}\right) .
$$

We choose $q:=R-R_{\delta}$ and

$$
\alpha:=\frac{\log 2}{\log 2+\log (R+q)}
$$

and arrive at

$$
(d(z))^{\alpha} \geq \frac{q^{\alpha}}{2^{k}} \quad\left(z \in U_{k}\right) .
$$

Finally, putting (6.1) and (6.2) together we get

$$
g(z) \leq \frac{\Gamma}{q^{\alpha}}(d(z))^{\alpha} \quad\left(z \in U_{k} \backslash U_{k-1}\right)
$$

which completes the proof.

Gehring and Väisälä [GV] have shown that quasicircles always have Hausdorff dimension less than two and thus by Corollary 4.5 we obtain:

Corollary 6.2. Let $\delta<\frac{1}{4}$ and $\left(c_{n}\right) \in K_{\delta}^{\mathbb{N}}$. Then $\operatorname{dim}_{\mathrm{H}} \mathcal{\partial}_{\left(c_{n}\right)}<2$.

If $0<\delta \leq \frac{1}{4}$ and $\left(c_{n}\right) \in K_{\delta}^{\mathbb{N}}$, then the Julia set $\mathcal{J}_{\left(c_{n}\right)}$ is connected so that its Hausdorff dimension is at least one. Moreover, Sullivan [Su] has shown, that if $c \neq 0$ is in the interior of the main cardioid of the Mandelbrot set, then $\operatorname{dim}_{\mathrm{H}} \mathcal{\partial}\left(f_{c}\right)>1$. Furthermore, it follows by a result of Shishikura [Sh] that $\operatorname{dim}_{\mathrm{H}} \mathcal{\partial}\left(f_{1 / 4}\right)=2$. It would be of interest whether $\operatorname{dim}_{\mathrm{H}} \mathcal{J}_{\left(c_{n}\right)}$ is almost surely (in the sense of Section 5) greater than one if $\left(c_{n}\right) \in K_{\delta}^{\mathbb{N}}$ for some $\delta<\frac{1}{4}$. In our general setting, it is clear that we can only expect such an almost surely statement. 


\section{Density of repelling fixpoints.}

From iteration theory of a fixed rational function it is well-known that the repelling periodic points are dense in the Julia set (cf. [Be, p. 148], [CG, p. 63] or [St, p. 35]). In our setting we consider the set $\mathcal{R}_{\left(c_{n}\right)}$ of repelling fixpoints of the sequence of iterates $\left(F_{n}\right)$, i.e.,

$$
\mathcal{R}_{\left(c_{n}\right)}:=\left\{\zeta \in \mathbb{C}: F_{k}(\zeta)=\zeta \text { for some } k \in \mathbb{N} \text { and }\left|F_{k}^{\prime}(\zeta)\right|>1\right\} .
$$

It is not necessarily true that $\mathcal{R}_{\left(c_{n}\right)} \subset \mathcal{J}_{\left(c_{n}\right)}$. But from a result of Fornæss and Sibony [FS, Theorem 2.3] it follows that if $\delta>0$ is sufficiently small and $\left(c_{n}\right) \in K_{\delta}^{\mathbb{N}}$, then $\left(\mathcal{R}_{\left(c_{n}\right)}\right)^{\prime}=\mathcal{J}_{\left(c_{n}\right)}$. More precisely, we show:

Theorem 7.1. Let $\delta<\frac{1}{4}$ and $\left(c_{n}\right) \in K_{\delta}^{\mathbb{N}}$. Then $\left(\mathcal{R}_{\left(c_{n}\right)}\right)^{\prime}=\mathcal{J}_{\left(c_{n}\right)}$.

Proof. Since $\delta<\frac{1}{4}$ we have $f_{c}\left(\bar{D}_{r}\right) \subset D_{r}$ for all $c \in K_{\delta}$ and $s_{\delta}<r<$ $r_{\delta}$. This implies that $F_{k}(z) \neq z$ for all $k \in \mathbb{N}$ and $s_{\delta}<|z|<r_{\delta}$. Since $F_{k}^{\prime}(z)=2^{k} \prod_{j=0}^{k-1} F_{j}(z)$ and $f_{c}\left(K_{1 / 2}\right) \subset K_{1 / 2}$, we have $\mathcal{R}_{\left(c_{n}\right)} \cap K_{1 / 2}=\varnothing$. Setting $K:=K_{r}$ for some $r \in\left(\frac{1}{2}, r_{\delta}\right)$, we also have $\mathcal{R}_{\left(c_{n}\right)} \cap K=\varnothing$. We set $U:=\mathbb{C} \backslash K$. If $z \in U$ and $F_{j}(z) \in U$ for all $j=1, \ldots, k-1$, then $\left|F_{k}^{\prime}(z)\right| \geq q^{k}$ with $q:=2 r>1$.

We first show that $\left(\mathcal{R}_{\left(c_{n}\right)}\right)^{\prime} \subset \mathcal{J}_{\left(c_{n}\right)}$. For that purpose let $F_{k_{\ell}}\left(z_{\ell}\right)=z_{\ell}$, $\left|F_{k_{\ell}}^{\prime}\left(z_{\ell}\right)\right|>1$ and $z_{\ell} \rightarrow \zeta$ as $\ell \rightarrow \infty$. If $\zeta \in \mathbb{C} \backslash \mathcal{K}_{\left(c_{n}\right)}$, then $F_{k_{\ell}} \rightarrow \infty$ as $\ell \rightarrow \infty$ uniformly in some neighbourhood of $\zeta$. This gives $F_{k_{\ell}}\left(z_{\ell}\right) \rightarrow \infty$ as $\ell \rightarrow \infty$ which is a contradiction. Now, assume that $\zeta \in\left(\mathcal{K}_{\left(c_{n}\right)}\right)^{\circ}$. If $F_{j}(\zeta) \in U$ for all $j \in \mathbb{N}_{0}$, then $\left|F_{k}^{\prime}(\zeta)\right| \geq q^{k} \rightarrow \infty$ as $k \rightarrow \infty$. But this is impossible since $\left(F_{k}\right)$ is normal and bounded in $\left(\mathcal{K}_{\left(c_{n}\right)}\right)^{\circ}$. Therefore, we have $F_{k_{0}}(\zeta) \in K$ for some $k_{0} \in \mathbb{N}_{0}$, and thus $F_{k}(\zeta) \in K$ for all $k \geq k_{0}$. By passing to a subsequence we may assume that $F_{k_{\ell}} \rightarrow \phi$ as $\ell \rightarrow \infty$ uniformly in some neighbourhood $U_{\zeta}$ of $\zeta$, where $\phi$ is holomorphic in $U_{\zeta}$. This implies $z_{\ell}=F_{k_{\ell}}\left(z_{\ell}\right) \rightarrow \phi(\zeta)$ as $\ell \rightarrow \infty$ and thus $z_{\ell} \in K$ for all $\ell$ large enough which is again a contradiction.

Now, we show that $\mathcal{J}_{\left(c_{n}\right)} \subset\left(\mathcal{R}_{\left(c_{n}\right)}\right)^{\prime}$. Suppose that there exists $\zeta \in \mathcal{J}_{\left(c_{n}\right)}$ and a neighbourhood $V$ of $\zeta$ such that $F_{k}(z) \neq z$ for all $z \in V$ and $k \geq k_{0}=$ $k_{0}(V)$. We set

$$
h_{k}(z):=\frac{1}{2^{k}} \log \left|F_{k}(z)-z\right| .
$$

Then $h_{k}$ is harmonic and uniformly bounded above in $V$. By Eq. (2.1) we have $h_{k} \rightarrow g_{\left(c_{n}\right)}$ as $k \rightarrow \infty$ in $V \backslash \mathcal{K}_{\left(c_{n}\right)}$, and thus $h_{k} \rightarrow h$ as $k \rightarrow \infty$ for some harmonic function $h$ in $V$. Furthermore, there holds $h_{k} \rightarrow 0$ as $k \rightarrow \infty$ in $V \cap \mathcal{K}_{\left(c_{n}\right)}$ so that $h=0$ in $V \cap \mathcal{K}_{\left(c_{n}\right)}$. But this is a contradiction to the minimum principle for harmonic functions.

Therefore, for every $\zeta \in \mathcal{J}_{\left(c_{n}\right)}$ there exists a strictly increasing sequence $\left(k_{\ell}\right)$ in $\mathbb{N}$ and $z_{\ell} \in U$ such that $z_{\ell} \rightarrow \zeta$ as $\ell \rightarrow \infty$ and $F_{k_{\ell}}\left(z_{\ell}\right)=z_{\ell}$. Then we 
have $F_{j}\left(z_{\ell}\right) \in U$ for $j=1, \ldots, k_{\ell}-1$ which gives $\left|F_{k_{\ell}}^{\prime}\left(z_{\ell}\right)\right| \geq q^{k_{\ell}}>1$ so that $z_{\ell} \in \mathcal{R}_{\left(c_{n}\right)}$.

It would be of interest whether Theorem 7.1 holds for all $\delta>0$. However, the proof shows that we always have $\left(\mathcal{R}_{\left(c_{n}\right)}\right)^{\prime} \subset \mathcal{K}_{\left(c_{n}\right)}$.

\section{Asymptotic distribution of predecessors.}

If $\left(c_{n}\right) \in K_{\delta}^{\mathbb{N}}$ and if $a \in \Delta_{R_{\delta}}$, then the predecessors $F_{k}^{-1}(a)$ of $a$ are all contained in $\mathcal{A}_{\left(c_{n}\right)}(\infty)$, and they only accumulate on the Julia set $\mathcal{J}_{\left(c_{n}\right)}$. In fact, this follows from the invariance of $\Delta_{R}$ for $R>R_{\delta}$ and $F_{k} \rightarrow \infty$ as $k \rightarrow \infty$ locally uniformly in $\mathcal{A}_{\left(c_{n}\right)}(\infty)$. We want to study the asymptotic distribution of $F_{k}^{-1}(a)$ as $k \rightarrow \infty$. For iteration of a fixed polynomial this was done by Brolin [Bro].

We first recall some facts from potential theory which are needed in the sequel and which can be found, for example, in the book of Tsuji $[\mathbf{T}]$. Let $E \subset \mathbb{C}$ be an infinite compact set, and let $D$ be its outer domain, that is the component of $\widehat{\mathbb{C}} \backslash E$ containing the point $\infty$. Furthermore, we denote by cap $E \geq 0$ the logarithmic capacity (or transfinite diameter) of $E$. (We do not recall the definition of cap $E$ because it will not be needed.) We suppose that the Green function $g_{D}$ of $D$ with pole at infinity exists. Then

$$
g_{D}(z)=\log |z|+V+o(1) \quad \text { as } z \rightarrow \infty
$$

and cap $E=e^{-V}>0$. Note that by Eq. (2.1) this is true for $E=\mathcal{J}_{\left(c_{n}\right)}$ with cap $E=1$. Now, let $\mu$ be any probability measure on $E$. Then the energy integral

$$
I[\mu]:=\iint_{E \times E} \log \frac{1}{|\zeta-\omega|} d \mu(\zeta) d \mu(\omega)
$$

is finite, and the logarithmic potential

$$
p_{\mu}(z):=\int_{E} \log \frac{1}{|z-\zeta|} d \mu(\zeta)
$$

is harmonic in $D$. Furthermore, there exists a unique probability measure $\mu^{*}$ on $E$ which minimizes the energy integral $I[\mu]$, and there holds

$$
g_{D}(z)-V=-p_{\mu^{*}}(z) \quad(z \in D) .
$$

This measure $\mu^{*}$ is called the equilibrium measure on $E$. In the following $\mu^{*}$ always denotes the equilibrium measure on the Julia set $\partial_{\left(c_{n}\right)}$, and $\operatorname{supp} \mu^{*}$ denotes its support, that is the set of points $z \in \mathcal{J}_{\left(c_{n}\right)}$ such that $\mu^{*}\left(D_{\varepsilon}(z) \cap\right.$ $\left.\partial_{\left(c_{n}\right)}\right)>0$ for every $\varepsilon>0$. Note that $\operatorname{supp} \mu^{*}$ is a closed set.

In order to study the asymptotic distribution of $F_{k}^{-1}(a)$ for $a \in \Delta_{R_{\delta}}$ as $k \rightarrow \infty$ we consider the following sequence $\left(\mu_{k}^{a}\right)$ of probability measures. 
If $\delta_{z}$ denotes the Dirac measure concentrated at the point $z \in \mathbb{C}$ (that is $\delta_{z}(E)=1$ if $z \in E$ and $\delta_{z}(E)=0$ if $\left.z \notin E\right)$, then let

$$
\mu_{k}^{a}:=\frac{1}{2^{k}} \sum_{F_{k}(z)=a} \delta_{z} .
$$

We will show that $\left(\mu_{k}^{a}\right)$ is weakly convergent to $\mu^{*}$, that is $\mu_{k}^{a}(E) \rightarrow \mu^{*}(E)$ as $k \rightarrow \infty$ for every Borel set $E \subset \mathbb{C}$ with $\mu^{*}\left(E^{\circ}\right)=\mu^{*}(\bar{E})$. For that purpose we first collect some auxiliary results.

Lemma 8.1 ([Bro, Lemma 15.4]). Let $E \subset \mathbb{C}$ be a compact set, and let $f$ be a function defined on $E$ such that for some constant $L$ there holds $\left|f\left(z_{1}\right)-f\left(z_{2}\right)\right| \leq L\left|z_{1}-z_{2}\right|$ for all $z_{1}, z_{2} \in E$. If cap $E=0$, then cap $f(E)=$ 0 .

Lemma 8.2. Let $\delta>0$ and $\left(c_{n}\right) \in K_{\delta}^{\mathbb{N}}$. Then $\operatorname{cap}\left(\mathcal{J}_{\left(c_{n}\right)} \backslash \operatorname{supp} \mu^{*}\right)=0$.

Proof. Since $\mathcal{J}_{\left(c_{n}\right)}=\partial \mathcal{A}_{\left(c_{n}\right)}(\infty)$ and cap $\mathcal{J}_{\left(c_{n}\right)}>0$, the assertion immediately follows from Theorem III.31 in [T, p. 79].

Lemma 8.3. Let $\delta>0$ and $\left(c_{n}\right) \in K_{\delta}^{\mathbb{N}}$. Then $\operatorname{supp} \mu^{*}=\mathcal{J}_{\left(c_{n}\right)}$.

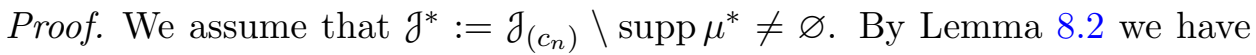
$\operatorname{cap} \mathcal{J}^{*}=0$. Since $\mathcal{J}^{*}$ is an open set in $\mathcal{J}_{\left(c_{n}\right)}$ we may choose $z_{0} \in \mathcal{J}^{*}$ and $\varepsilon>0$ such that $\partial_{\varepsilon}:=\partial^{*} \cap D_{\varepsilon}\left(z_{0}\right) \subset \partial^{*}$. We also have cap $\partial_{\varepsilon}=0$. But by the self-similarity of $\mathcal{J}_{\left(c_{n}\right)}$ (cf. [Bü1]) there exists $m \in \mathbb{N}$ such that $F_{m}\left(\mathcal{J}_{\varepsilon}\right)=F_{m}\left(\mathcal{J}_{\left(c_{n}\right)}\right)$. Since $\left|f_{c_{k}}\left(z_{1}\right)-f_{c_{k}}\left(z_{2}\right)\right|=\left|z_{1}+z_{2}\right|\left|z_{1}-z_{2}\right| \leq 2 R_{\delta}\left|z_{1}-z_{2}\right|$ for all $k \in \mathbb{N}$ and $z_{1}, z_{2} \in \mathcal{J}_{\left(c_{n}\right)}$, we obtain cap $F_{m}\left(\mathcal{J}_{\varepsilon}\right)=0$ by Lemma 8.1. On the other hand there holds $F_{m}\left(\mathcal{J}_{\left(c_{n}\right)}\right)=\mathcal{J}_{\left(c_{n+m}\right)}$ and thus cap $F_{m}\left(\mathcal{J}_{\left(c_{n}\right)}\right)=1$ which gives a contradiction.

Lemma 8.4 ([Bro, Lemma 15.5]). Let $E, H \subset \mathbb{C}$ be compact sets with $E \subset$ $H$ and cap $E=e^{-V}>0$. Furthermore, let $\left(\mu_{n}\right)$ be a sequence of probability measures on $H$ which converges weakly to a probability measure $\mu$ on $E$. If $u_{n}$ denotes the logarithmic potential with respect to $\mu_{n}$ and $\mu^{*}$ denotes the equilibrium measure on $E$, then suppose $\liminf _{n \rightarrow \infty} u_{n}(z) \geq V$ for $z \in E$ and $\operatorname{supp} \mu^{*}=E$. Then there holds $\mu=\mu^{*}$.

Theorem 8.5. Let $\delta>0$ and $\left(c_{n}\right) \in K_{\delta}^{\mathbb{N}}$. Then for any $a \in \Delta_{R_{\delta}}$ the sequence $\left(\mu_{k}^{a}\right)$ of probability measures defined by (8.1) converges weakly to the equilibrium measure $\mu^{*}$ on $\mathcal{J}_{\left(c_{n}\right)}$.

Proof. For $k \in \mathbb{N}$ let $z_{1, k}, \ldots, z_{2^{k}, k}$ be the solutions of the equation $F_{k}(z)=a$. Then we have $z_{j, k} \in \mathcal{A}_{\left(c_{n}\right)}(\infty)$ and $z_{j, k} \in H:=K_{|a|}$ for $j=1, \ldots, 2^{k}$ so that $\operatorname{supp} \mu_{k}^{a} \subset H$. Since $\left|F_{k}(z)\right| \leq R_{\delta}$ for $z \in \mathcal{J}_{\left(c_{n}\right)}$ and

$$
\left|F_{k}(z)-a\right|=\prod_{j=1}^{2^{k}}\left|z-z_{j, k}\right|,
$$


we obtain for $z \in \mathcal{J}_{\left(c_{n}\right)}$

$$
\sum_{j=1}^{2^{k}} \log \left|z-z_{j, k}\right|=\log \left|F_{k}(z)-a\right| \leq \log \left(R_{\delta}+|a|\right)=C
$$

and thus

$$
u_{k}(z):=\frac{1}{2^{k}} \sum_{j=1}^{2^{k}} \log \frac{1}{\left|z-z_{j, k}\right|} \geq-\frac{C}{2^{k}}
$$

This can be written as

$$
u_{k}(z)=\int_{H} \log \frac{1}{|z-\zeta|} d \mu_{k}^{a}(\zeta) \geq-\frac{C}{2^{k}}
$$

so that

$$
\liminf _{k \rightarrow \infty} u_{k}(z) \geq 0=\log \operatorname{cap} \mathcal{J}_{\left(c_{n}\right)} \quad\left(z \in \mathcal{J}_{\left(c_{n}\right)}\right) .
$$

By the Selection Theorem (cf. [T, p. 34]) every sequence of probability measures on $H$ contains a weakly convergent subsequence. Therefore, we only have to show that for every subsequence of $\left(\mu_{k}^{a}\right)$ which converges weakly to some probability measure $\nu$ there holds $\nu=\mu^{*}$. In fact, since the predecessors $F_{k}^{-1}(a)$ of $a$ do not accumulate in $\mathcal{A}_{\left(c_{n}\right)}(\infty)$ we obtain $\operatorname{supp} \nu \subset \mathcal{J}_{\left(c_{n}\right)}$, and because of (8.2) the assertion follows from Lemma 8.3 and 8.4.

Remark 8.6. If $\delta<\frac{1}{4}$ and $\left(c_{n}\right) \in K_{\delta}^{\mathbb{N}}$, then the assertion of Theorem 8.5 also holds for any $a \in D_{r_{\delta}}$. This requires only a few simple modifications in the proof.

Like in the iteration of a fixed function there holds that for any $a \in \mathcal{J}_{\left(c_{n}\right)}$ the set $\bigcup_{k=1}^{\infty} F_{k}^{-1}\left(F_{k}(a)\right)$ is dense in $\mathcal{J}_{\left(c_{n}\right)}$ (cf. [Bü1]). We also want to study the asymptotic distribution of $F_{k}^{-1}\left(F_{k}(a)\right)$ as $k \rightarrow \infty$. For that purpose, we consider the following sequence $\left(\nu_{k}^{a}\right)$ of probability measures defined by

$$
\nu_{k}^{a}:=\frac{1}{2^{k}} \sum_{F_{k}(z)=F_{k}(a)} \delta_{z} .
$$

Then $\operatorname{supp} \nu_{k}^{a} \subset \mathcal{J}_{\left(c_{n}\right)}$, and from iteration theory of a fixed polynomial $f_{c}$ it is known (cf. [Bro], see also [St, p. 148]) that $\left(\nu_{k}^{a}\right)$ converges weakly to the equilibrium measure $\mu^{*}$ on $\mathcal{J}\left(f_{c}\right)$. We show that this holds true in our situation. 
Theorem 8.7. Let $\delta>0$ and $\left(c_{n}\right) \in K_{\delta}^{\mathbb{N}}$. Then for any $a \in \mathcal{J}_{\left(c_{n}\right)}$ the sequence $\left(\nu_{k}^{a}\right)$ of probability measures defined by (8.3) converges weakly to the equilibrium measure $\mu^{*}$ on $\mathrm{J}_{\left(c_{n}\right)}$.

Proof. For $k \in \mathbb{N}$ let $z_{1, k}, \ldots, z_{2^{k}, k}$ be the solutions of the equation $F_{k}(z)=$ $F_{k}(a)$. Then we have for $z \in \mathcal{A}_{\left(c_{n}\right)}(\infty)$

$$
\frac{1}{2^{k}} \log \left|F_{k}(z)-F_{k}(a)\right|=\frac{1}{2^{k}} \sum_{j=1}^{2^{k}} \log \left|z-z_{j, k}\right|=\int_{\mathcal{J}_{\left(c_{n}\right)}} \log |z-\zeta| d \nu_{k}^{a}(\zeta) .
$$

Again, we only have to show that every weakly convergent subsequence $\left(\lambda_{\ell}\right)$ of $\left(\nu_{k}^{a}\right)$ has the limit $\mu^{*}$. If $\lambda_{\ell} \rightarrow \lambda$ as $\ell \rightarrow \infty$ weakly, then for $z \in$ $\mathcal{A}_{\left(c_{n}\right)}(\infty)$

$$
\lim _{\ell \rightarrow \infty} \int_{\mathcal{J}_{\left(c_{n}\right)}} \log |z-\zeta| d \lambda_{\ell}(\zeta)=\int_{\mathcal{J}_{\left(c_{n}\right)}} \log |z-\zeta| d \lambda(\zeta) .
$$

On the other hand we have

$$
\begin{aligned}
\frac{1}{2^{k}} \log \left|F_{k}(z)-F_{k}(a)\right|= & \frac{1}{2^{k}} \log \left|\frac{F_{k}(z)-F_{k}(a)}{F_{k}(z)}\right| \\
& +\frac{1}{2^{k}} \log \left|F_{k}(z)\right| \rightarrow g_{\left(c_{n}\right)}(z) \quad \text { as } k \rightarrow \infty .
\end{aligned}
$$

This implies

$$
g_{\left(c_{n}\right)}(z)=\int_{\mathcal{J}_{\left(c_{n}\right)}} \log |z-\zeta| d \lambda(\zeta) \quad\left(z \in \mathcal{A}_{\left(c_{n}\right)}(\infty)\right),
$$

and since $\mu^{*}$ is unique the assertion follows.

\section{References}

[A] L.V. Ahlfors, Lectures on Quasiconformal Mappings, van Nostrand, Princeton, 1966 .

[Be] A.F. Beardon, Iteration of Rational Functions, Springer-Verlag, New York, 1991.

[BeP] A.F. Beardon and Ch. Pommerenke, The Poincaré metric of plane domains, J. London Math. Soc. (2), 41 (1979), 475-483.

[Bl] P. Blanchard, Complex analytic dynamics on the Riemann sphere, Bull. Amer. Math. Soc. (N.S.), 11 (1984), 85-141.

[Bro] H. Brolin, Invariant sets under iteration of rational functions, Ark. Mat., 6 (1965), 103-144.

[Brü] R. Brück, Connectedness and stability of Julia sets of the composition of polynomials of the form $z^{2}+c_{n}$, J. London Math. Soc. (2), 61 (2000), 462-470.

[BBR] R. Brück, M. Büger and S. Reitz, Random iterations of polynomials of the form $z^{2}+c_{n}$ : Connectedness of Julia sets, Ergodic Theory Dynam. Systems, 19 (1999), 1221-1231. 
[Bü1] M. Büger, Self-similarity of Julia sets of the composition of polynomials, Ergodic Theory Dynam. Systems, 17 (1997), 1289-1297.

[Bü2] - On the composition of polynomials of the form $z^{2}+c_{n}$, Math. Ann., 310 (1998), 661-683.

[C] L. Carleson, Removable singularities of continuous harmonic functions in $\mathbb{R}^{m}$, Math. Scand., 12 (1963), 15-18.

[CG] L. Carleson and T.W. Gamelin, Complex Dynamics, Springer-Verlag, New York, 1993.

[EL] A.E. Eremenko and M.Yu. Lyubich, The dynamics of analytic transformations, Algebra i Analiz, 1 (1989), 1-70; English transl. in Leningrad Math. J., 1 (1990), 563-634.

[FS] J.E. Fornæss and N. Sibony, Random iterations of rational functions, Ergodic Theory Dynam. Systems, 11 (1991), 687-708.

[G] V. Garber, On the iteration of rational functions, Math. Proc. Cambridge Philos. Soc., 84 (1978), 497-505.

[GV] F.W. Gehring and J. Väisälä, Hausdorff dimension and quasiconformal mappings, J. London Math. Soc. (2), 6 (1973), 504-512.

$[\mathrm{LV}]$ O. Lehto and K.I. Virtanen, Quasiconformal Mappings in the Plane, SpringerVerlag, Berlin, 1973.

[L1] M.Yu. Lyubich, On typical behavior of the trajectories of a rational mapping of the sphere, Dokl. Akad. Nauk SSSR, 268 (1983), 29-32; English transl. in Soviet Math. Dokl., 27 (1983), 22-25.

[L2] The dynamics of rational transforms: The topological picture, Uspekhi Mat. Nauk, 41 (1986), 35-95; English transl. in Russian Math. Surveys, 41 (1986), 43-117.

[MR] R. Mañé and L.F. da Rocha, Julia sets are uniformly perfect, Proc. Amer. Math. Soc., 116 (1992), 251-257.

[M] J. Milnor, Dynamics in One Complex Variable, Vieweg Verlag, Braunschweig, 1999.

[NV] R. Näkki and J. Väisälä, John disks, Exposition. Math., 9 (1991), 3-43.

[P1] Ch. Pommerenke, Uniformly perfect sets and the Poincaré metric, Arch. Math. (Basel), 32 (1979), 192-199.

[P2] B Boundary Behaviour of Conformal Maps, Springer-Verlag, Berlin, 1992.

[Sh] M. Shishikura, The Hausdorff dimension of the boundary of the Mandelbrot set and Julia sets, Ann. of Math. (2), 147 (1998), 225-267.

[St] N. Steinmetz, Rational Iteration (Complex Analytic Dynamical Systems), Walter de Gruyter, Berlin, 1993.

[Su] D. Sullivan, Conformal dynamical systems, in 'Geometric Dynamics' (J. Palis, editor), Lecture Notes in Mathematics, 1007, Springer-Verlag, New York, 1983, 725-752.

[T] M. Tsuji, Potential Theory in Modern Function Theory, Maruzen, Tokyo, 1959. 
[Y] M.V. Yakobson, The boundaries of certain normality domains for rational maps, Uspekhi Mat. Nauk, 39 (1984), 211-212; English transl. in Russian Math. Surveys, 39 (1984), 229-230.

Received June 16, 1999, and revised September 20, 1999. The main parts of this research work were done during a temporary stay of the author at Fachbereich 7 - Mathematik of Bergische Universität - Gesamthochschule Wuppertal.

Justus-Liebig-Universität Giessen

ARndTstrasse 2

35392 GIESSEN

Germany

E-mail address: rainer.brueck@math.uni-giessen.de 